medRxiv preprint doi: https://doi.org/10.1101/2020.07.22.20159855; this version posted July 24, 2020. The copyright holder for this preprint (which was not certified by peer review) is the author/funder, who has granted medRxiv a license to display the preprint in perpetuity.

It is made available under a CC-BY-NC-ND 4.0 International license .

\title{
Renin-Angiotensin-Aldosterone-System inhibitor use in patients with COVID-19 infection and prevention of serious events: a cohort study in commercially insured patients in the US
}

\author{
Maria C Schneeweiss, MD ${ }^{1,2)}$ \\ Sandra Leonard, $\mathrm{MPH}^{3)}$ \\ Andrew Weckstein ${ }^{4)}$ \\ Sebastian Schneeweiss, MD, ScD ${ }^{1,2)}$ \\ Jeremy A Rassen, ScD ${ }^{4)}$
}

July 22,2020

1) Division of Pharmacoepidemiology and Pharmacoeconomics, Department of Medicine, and Department of Dermatology Brigham and Women's Hospital, Boston, MA

2) Harvard Medical School, Boston, MA

3) HealthVerity, Inc.

4) Aetion, Inc.

Correspondence to:

Maria Schneeweiss, MD

Division of Pharmacoepidemiology and Pharmacoeconomics, Department of Medicine, Brigham and Women's Hospital

1 Brigham Circle, suite 3030, Boston, MA 02120; Phone: 617 278-0930

Email: mschneeweiss@bwh.harvard.edu

Running head: ACEi/ARB use and severity COVID-19 infection

Manuscript: 303 words (abstract); 2020 words (text); 1 figure; 4 tables

Supplemental materials: Appendix

Funding/Support: No further funding was received for this study.

\section{Financial Disclosure:}

All authors have completed the Unified Competing Interest form (available on request from the corresponding author) and declare:

Dr. Schneeweiss M. has no relevant financial interests to report.

Ms. Leonard is employee of HealthVerity Inc. and holds equity

Dr. Schneeweiss $S$. is the principal investigator of investigator-initiated grants to the Brigham and

Women's Hospital from the FDA, NIH, PCORI, Bayer, Vertex, and Boehringer Ingelheim unrelated to 
medRxiv preprint doi: https://doi.org/10.1101/2020.07.22.20159855; this version posted July 24, 2020. The copyright holder for this preprint (which was not certified by peer review) is the author/funder, who has granted medRxiv a license to display the preprint in perpetuity. It is made available under a CC-BY-NC-ND 4.0 International license.

the topic of this study. He is a consultant to Aetion, a software manufacturer of which he owns equity. His interests were declared, reviewed, and approved by the Brigham and Women's Hospital and Partners HealthCare System in accordance with their institutional compliance policies.

Drs. Weckstein and Rassen are employees of Aetion Inc. and hold equity. 
medRxiv preprint doi: https://doi.org/10.1101/2020.07.22.20159855; this version posted July 24, 2020. The copyright holder for this preprint (which was not certified by peer review) is the author/funder, who has granted medRxiv a license to display the preprint in perpetuity.

It is made available under a CC-BY-NC-ND 4.0 International license .

\section{Abstract}

Objectives: There is lack of clarity regarding the role of angiotensin receptor blockers (ARB) or angiotensin converting enzyme inhibitors (ACEi) in interfering with the SARS-COV-2 binding on human cells and the resulting change in disease severity. We sought to assess the risk of hospitalization for COVID-19 and serious complications in current users of ARB or ACEi compared to users of dihydropyridine calcium channel blockers (dhpCCB).

Design: Cohort study

Setting: The analysis used de-identified, patient level data from HealthVerity, linking longitudinal data from US medical and pharmacy claims, which contain information on inpatient or outpatient diagnoses, procedures and medication dispensing.

Participants: We identified patients aged 40+ and free of chronic kidney disease (CKD) who were newly diagnosed COVID-19, between March 1, 2020 and May 30, 2020, and adherent to ACEi, ARB, or dhpCCB therapy.

Interventions: Current use of an $\mathrm{ACEi}, \mathrm{ARB}$, or dhpCCB.

Main outcome measures: We compared the 30-day risk of hospitalization for COVID-19 and serious complications.

Results: Of 24,708 patients identified, 7,571 were current users of an ARB, 8,484 of an ACEi, and 8,653 of a dhpCCB. The unadjusted 30-day risk of hospitalization for COVID-19 was $2.66 \%$ among ARB users, and $2.90 \%$ among ACEi users and $3.68 \%$ in dhpCCB users. In the PS-matched cohort, the risk of hospitalization among $A R B$ users was $17 \%$ lower as compared to dhpCCB $(R R=0.83 ; 0.68$ $1.00)$, and the risk among $A C E$ users was $10 \%$ lower as compared to dhpCCB (RR=0.90; 0.76-1.07). When including patients with pre-existing $C K D$, the protective effect of $A R B(R R=0.74 ; 0.62-0.88$ ) and ACEi (RR=0.84; 0.71-0.99) was more pronounced. 
medRxiv preprint doi: https://doi.org/10.1101/2020.07.22.20159855; this version posted July 24, 2020. The copyright holder for this preprint (which was not certified by peer review) is the author/funder, who has granted medRxiv a license to display the preprint in perpetuity. It is made available under a CC-BY-NC-ND 4.0 International license .

Conclusions: This cohort study showed that neither ARB nor ACEi use increase the risk of severe COVID-19 disease among those infected, and instead suggests that current use of ARB may offer a protective effect. This study found no evidence to support the discontinuation of ARB/ACEi therapy. 
medRxiv preprint doi: https://doi.org/10.1101/2020.07.22.20159855; this version posted July 24, 2020. The copyright holder for this preprint (which was not certified by peer review) is the author/funder, who has granted medRxiv a license to display the preprint in perpetuity. It is made available under a CC-BY-NC-ND 4.0 International license .

\section{Background}

The spike proteins covering SARS-COV-2 bind to angiotensin-converting enzyme-2 (ACE2) receptors primarily on type II alveolar cells and cells in the heart, kidney, liver, and gastrointestinal tract, enabling the virus to inject its RNA. ${ }^{1}$ It has been hypothesized that blocking the ACE2 receptor may decrease SARS-COV-2's ability to bind to human cells, and thus reduce the severity of COVID-19 illness. ${ }^{1}$ However, there is uncertainty surrounding the effect of renin-angiotensin-aldosterone system (RAAS) inhibition in patients with COVID-19, with some hypothesizing that angiotensin receptor blockers (ARB) or angiotensin converting enzyme inhibitors (ACEi) use may increase the propagation of the virus. ${ }^{1-4}$

Any link between ACEi or ARB use and the virus RNA's ability to enter human cells could yield an effect, positive or negative, on the clinical severity of a COVID-19 infection, including the need for hospitalization, ventilator use, intensive care unit (ICU) stay, or death. Pre-published cohort studies by Giorgi, Mehta, and Rentsch examined the use of ACEi/ARB with respect to hospital admissions and found an increased risk; however, the contrast they made compared ACEi/ARB users to nonusers and likely did not fully account for underlying factors associated with both the need for ACE or ARB treatment and with poor health outcomes. ${ }^{5-7}$ Khera et al. addressed some of these issues by equalizing risk through requiring comparator patients to have hypertension and to be treated with a guideline-recommended antihypertensive, and found a protective effect with ACEi use, but not ARB use, among Medicare beneficiaries. ${ }^{8}$ The literature lacks study findings examining the use of ACEi or ARB therapy across a broad population, and the associated risk of severe outcomes from COVID19. ${ }^{4}$ As such, clinicians lack clear guidance on appropriate action to take with patients using an ACEi or ARB and diagnosed with COVID-19, in particular whether to discontinue therapy. 
medRxiv preprint doi: https://doi.org/10.1101/2020.07.22.20159855; this version posted July 24, 2020. The copyright holder for this preprint (which was not certified by peer review) is the author/funder, who has granted medRxiv a license to display the preprint in perpetuity.

It is made available under a CC-BY-NC-ND 4.0 International license .

To that end, this study compares the risk of hospitalization for COVID-19 disease and hospitalization for serious complications of COVID-19 among prevalent users of ACEi or ARB as compared with prevalent users of dihydropyridine calcium channel blockers (dhpCCB), using recently-collected administrative claims data from the US.

\section{Methods}

\section{Data source}

This study used de-identified patient level healthcare data from HealthVerity covering $>100$ million unique patients with data from over 60 unique data sources in the US, including medical claims, pharmacy claims, lab, and electronic medical records (EMR). Data encompass all major US payer types, including commercial, Medicaid, and Medicare. For this study, we examined linked medical claims, pharmacy claims, and laboratory data containing diagnoses, procedures, prescriptions and other variables. Claims included both unadjudicated insurance claims available in near real-time time from practice management systems, billing systems, and claims clearinghouses which were then linked with adjudicated claims sourced from insurance providers and payers as well as laboratory data from hospital billing systems. Data for this study covered the period of December 1 , 2018 through May 30, 2020. Claims include dates of service and diagnoses, and are recorded longitudinally, thus allowing us to establish baseline health status, COVID-19 diagnosis, and any sequalae. This study was approved by the New England IRB (\#1-9757-1). De-identified patient level study data can be made available for inspection.

\section{$\underline{\text { Patients }}$}


medRxiv preprint doi: https://doi.org/10.1101/2020.07.22.20159855; this version posted July 24, 2020. The copyright holder for this preprint (which was not certified by peer review) is the author/funder, who has granted medRxiv a license to display the preprint in perpetuity.

It is made available under a CC-BY-NC-ND 4.0 International license .

Eligible patients had at least one medical encounter related to COVID-19. A COVID-19-related encounter was defined as any claim with an International Classification of Diseases, 10th Revision, Clinical Modification (ICD-10-CM) code in accordance with the Center for Disease Control's (CDC) interim (B34.2, B97.21, B97.29, J12.81) and final (U07.1) coding guidance for confirmed cases of COVID-19a or who had a positive or presumed positive COVID-19 viral lab test between March 1, 2020 and May 30, 2020 (see Appendix A for further details).

To compare the impact of ongoing ACEi or ARB therapy on severity of COVID-19 infection, we implemented a prevalent user, active comparator design where balance between treatment groups at baseline was achieved through study design and propensity score (PS) matching. ${ }^{9,10}$ A prevalent user design, where all study participants were using one of the study medications prior to the index date, was chosen in order to understand the effect of medications being taken at the time of COVID-19 infection, rather than the effect of initiation of therapy in response to COVID-19 diagnosis. To improve comparability among treatment groups, prevalent users of a dhpCCB were selected as the active comparator; these agents are also an anti-hypertensive treatment, like ACEi and ARB, yet due to their different mechanism of action, have no hypothesized effect on COVID-19.

We created 2 cohorts for analysis: cohort 1 included prevalent users of ARB versus dhpCCB, and cohort 2 included prevalent users of ACEi versus dhpCCB. Cohort entry was defined as the first outpatient visit with a COVID-19 diagnosis among patients with at least one prescription for an

\footnotetext{
${ }^{a}$ CDC. ICD-10-CM Official Coding and Reporting Guidelines. April 1, 2020 through September 30, 2020. https://www.cdc.gov/nchs/data/icd/COVID-19-guidelines-final.pdf.
} 
medRxiv preprint doi: https://doi.org/10.1101/2020.07.22.20159855; this version posted July 24, 2020. The copyright holder for this preprint (which was not certified by peer review) is the author/funder, who has granted medRxiv a license to display the preprint in perpetuity.

It is made available under a CC-BY-NC-ND 4.0 International license .

ACEi, ARB, or dhpCCB within the 90 days before cohort entry. We excluded patients if they: (1) had no other medical or pharmacy claims activity in the 90 days prior to cohort entry, (2) qualified for more than one treatment category, (3) were of age less than 40 years on cohort entry, or (4) had a diagnosis of chronic kidney disease (CKD) within the 90 days prior to cohort entry, as CKD is a contraindication for use of an ACEi or ARB (though not for dhpCCB). We conducted a sensitivity analysis that included patients with pre-existing CKD.

\section{$\underline{\text { Outcomes }}$}

We compared the risk of (1) hospitalization for COVID-19 and (2) hospitalization for serious COVID19 complications in the 30 days following cohort entry. We identified hospitalization for COVID-19 as the first inpatient visit with a diagnosis of COVID-19 (ICD-10 code: U07.1) following CDC-defined

outpatient diagnosis of COVID-19. We identified hospitalization for serious COVID-19 complications as the first inpatient visit with a diagnosis of COVID-19, as above, followed on the same day or within 30 days by a diagnosis code for acute organ dysfunction or failure, acute respiratory distress syndrome (ARDS), sepsis, or respiratory intubation or mechanical ventilation (see Appendix B for a full list of codes).

Follow-up started the day after cohort entry and continued until the earliest of occurrence of outcome, death, disenrollment, 30 days, or the end of data (May 30, 2020).

\section{Patient characteristics}

For each patient, we recorded age at cohort entry, sex, and month of cohort entry. Over the 90 days prior to cohort entry, we assessed: history of pneumonia infection requiring an office visit or 
medRxiv preprint doi: https://doi.org/10.1101/2020.07.22.20159855; this version posted July 24, 2020. The copyright holder for this preprint (which was not certified by peer review) is the author/funder, who has granted medRxiv a license to display the preprint in perpetuity.

It is made available under a CC-BY-NC-ND 4.0 International license .

hospitalization, prior use of asthma medications or anticoagulants, number of physician visits, number of unique prescription medications filled, and a range of comorbidities that may be risk factors for more severe COVID-19 disease (Table 2). Comorbidities included malignancy, alcohol use disorder, coagulation deficiency, HIV or AIDS, paralysis, valvular heart disease, weight loss, overweight or obesity, smoking, acute coronary syndrome, arrythmias, asthma, chronic pulmonary disease, conduction disorders, congestive heart failure, dependence on supplemental oxygen, hematologic disorders, hypertension, inflammatory heart disease, ischemic heart disease, liver disease, myocardial infarction, stroke, pulmonary circulation disorders, rheumatic disease, and systemic lupus erythematosus.

\section{$\underline{\text { Statistical analysis }}$}

We tabulated baseline patient characteristics and assessed the 30-day risk of the outcomes of interest with 95\% confidence intervals for each exposure group.

To account for potential confounding by measured patient characteristics, we used propensity score (PS)-matching to achieve balance of covariates between the treatment groups. ${ }^{11,12}$ While a prevalent user analysis can lead to bias from adjusting for intermediate variables, ${ }^{13}$ in this case both the drug exposure and the patient characteristics were observed before cohort entry, and thus are unlikely to cause bias. The PS, defined as the probability that a patient was using either ACEi/ARB versus dhpCCB, conditioning on all the confounders listed above, was estimated with a multivariable logistic regression model. PS-matching was performed using 1:1 nearest neighbor matching with a maximum matching caliper of 0.02 on the PS scale. ${ }^{14}$ To quantify balance achieved 
medRxiv preprint doi: https://doi.org/10.1101/2020.07.22.20159855; this version posted July 24, 2020. The copyright holder for this preprint (which was not certified by peer review) is the author/funder, who has granted medRxiv a license to display the preprint in perpetuity. It is made available under a CC-BY-NC-ND 4.0 International license .

at baseline, we computed standardized differences in the covariates between the two groups after PS-matching. ${ }^{15}$

We calculated relative risks (RR) and $95 \%$ confidence intervals (Cls) of the outcomes associated with drug use using logistic regression. All analyses were conducted using the Aetion Evidence Platform v3.19 (including R version 4.0.2), which has been validated and has successfully predicted randomized control trial findings. ${ }^{16-18}$ All analyses from the raw patient-level data are recorded with audit trails and can be reviewed on request.

\section{Results}

We identified 24,708 patients aged 40 years or older who had an outpatient diagnosis of COVID-19 and met the inclusion criteria (Table 1). At the start of follow-up, cohort 1 had 15,829 prevalent users of either an ARB $(n=7,571)$ or $\operatorname{dhpCCB}(n=8,258)$, while cohort 2 had 17,137 prevalent users of either an ACEi $(n=8,484)$ or dhpCCB $(n=8,653)$ (Tables 2, 3). After applying 1:1 PS-matching on all baseline characteristics, cohort 1 had 6,710 pairs of patients using ARB vs. dhpCCB and cohort 2 had 7,334 pairs of patients using ACEi vs. dhpCCB. In the PS-matched cohort, all 60 baseline characteristics were well-balanced between treatment groups (Tables 2, 3) with all absolute standardized differences between treatment groups below the recognized threshold of $0.1 .{ }^{19}$

In the PS-matched cohort, the 30-day risk of hospitalization for COVID-19 was $2.85 \%$ in ARB users (vs. 3.44\% in dhpCCB users) and 3.12\% in ACEi users (vs. 3.46\% in dhpCCB users) (Table 4). After PSmatching we found that ARB users had a 17\% decrease in the risk of hospitalization for COVID-19 $(\mathrm{RR}=0.83 ; 95 \% \mathrm{Cl}, 0.68-1.00)$ as compared to $\mathrm{dhpCCB}$, while $\mathrm{ACEi}$ users had a $10 \%$ decrease in risk 
medRxiv preprint doi: https://doi.org/10.1101/2020.07.22.20159855; this version posted July 24, 2020. The copyright holder for this preprint (which was not certified by peer review) is the author/funder, who has granted medRxiv a license to display the preprint in perpetuity. It is made available under a CC-BY-NC-ND 4.0 International license.

for hospitalization for COVID-19 (RR=0.90; 0.76-1.07) as compared to dhpCCB. Point estimates suggested protective effects for several serious complications, though confidence intervals generally included the null: acute organ dysfunction or failure ( $\mathrm{RR}=0.88[95 \% \mathrm{Cl}: 0.65-1.20]$ for $A R B$ and 1.00 [0.76-1.32] for ACEi); ARDS (RR=0.86 [0.63-1.16] for ARB and 0.99 [0.75-1.31] for ACEi); sepsis (RR=0.71 [0.49-1.03] for ARB and 1.06 [0.76-1.48] for ACEi); and for respiratory intubation or mechanical ventilation ( $R R=0.77$ [0.59-1.00] for ARB and 0.99 [0.78-1.26] for ACEi) (Table 4).

In our sensitivity analysis including patients with pre-existing CKD in the 90 days before outpatient COVID-19 diagnosis, the relative risk of hospitalization for COVID-19 decreased to $0.74(0.62-0.88)$ for ARB and 0.84 (0.71-0.99) for ACEi.

\section{Discussion}

In this population-representative study of patients aged 40 and older with an outpatient COVID-19 diagnosis, we observed a $17 \%$ decrease in the 30 -day risk of hospitalization for COVID-19 among ARB users compared to dhpCCB users, and a $10 \%$ decrease in risk of hospitalization for COVID-19 among ACEi users compared to dhpCCB users. We also observed a numerically decreased 30-day risk of respiratory intubation and mechanical ventilation after hospitalization with COVID-19 among ARB users compared to dhpCCB users. There was no meaningful difference in the risk of acute organ dysfunction, ARDS, or sepsis among RAAS inhibitors vs. dhpCCBs. 
medRxiv preprint doi: https://doi.org/10.1101/2020.07.22.20159855; this version posted July 24, 2020. The copyright holder for this preprint (which was not certified by peer review) is the author/funder, who has granted medRxiv a license to display the preprint in perpetuity.

It is made available under a CC-BY-NC-ND 4.0 International license .

Our findings suggest neither a need to discontinue ACEi/ARB therapy among patients using those medications at the time of COVID-19 diagnosis, nor a clear case for use of ACEi/ARB therapy as prophylaxis among COVID-19-infected patients.

There were several notable strengths of this study. First, we used a large population-representative cohort with over 20,000 COVID-19 adults 40+ receiving anti-hypertensive treatment with RAAS inhibitors or dhpCCB from established data sources. Second, the outcome of interest was severe enough to avoid differential surveillance bias since all serious infections that require hospitalization are captured in claims data, and the ICD codes used to identify them have been shown to be of high accuracy. ${ }^{20}$ Third, we implemented robust adjustment for potential confounders using active comparator study design combined with 1:1 propensity score matching. ${ }^{21,22}$ Fourth, by requiring comparator patients also be treated with an antihypertensive medication, the confounder-adjusted, dhpCCB control group provided an appropriate reference value for hospitalization in patients aged 40 or older with COVID-19 infection .

This population-based study applying a causal study design concludes that neither ARB nor ACEi use increase the risk of severe COVID-disease among those infected, and rather suggest that prevalent use of these medications - ARB in particular -- may offer a protective effect. Indeed, our findings suggest a small decrease in risk of hospitalization for COVID-19 between adult patients with an outpatient COVID-19 diagnosis who were using RAAS inhibitors versus those treated with dhpCCB. These findings are reassuring as they suggest that RAAS inhibitors should not be discontinued among patients with COVID-19 infection. 
medRxiv preprint doi: https://doi.org/10.1101/2020.07.22.20159855; this version posted July 24, 2020. The copyright holder for this preprint

(which was not certified by peer review) is the author/funder, who has granted medRxiv a license to display the preprint in perpetuity.

It is made available under a CC-BY-NC-ND 4.0 International license .

\section{References:}

1. Vaduganathan M, Vardeny O, Michel T, McMurray JJV, Pfeffer MA, Solomon SD. Renin-Angiotensin-Aldosterone System Inhibitors in Patients with Covid-19. N Engl J Med. 2020;382(17):1653-1659.

2. Khan A, Benthin C, Zeno B, et al. A pilot clinical trial of recombinant human angiotensin-converting enzyme 2 in acute respiratory distress syndrome. Crit Care. 2017;21(1):234.

3. South AM, Tomlinson L, Edmonston D, Hiremath S, Sparks MA. Controversies of reninangiotensin system inhibition during the COVID-19 pandemic. Nature reviews Nephrology. 2020;16(6):305-307.

4. Patel AB, Verma A. COVID-19 and Angiotensin-Converting Enzyme Inhibitors and Angiotensin Receptor Blockers: What Is the Evidence? JAMA. 2020.

5. Rossi P, G.,, Marino M, Formisano D, Venturelli F, Vicentini M, Grilli R. Characteristics and outcomes of a cohort of SARS-CoV-2 patients in the Province of Reggio Emilia, Italy. MedRxiv. 2020.

6. Mehta N, Kalra A, Nowacki AS, et al. Association of Use of Angiotensin-Converting Enzyme Inhibitors and Angiotensin II Receptor Blockers With Testing Positive for Coronavirus Disease 2019 (COVID-19). JAMA cardiology. 2020.

7. Christopher T. Rentsch, Farah Kidwai-Khan, Janet P. Tate, et al. Covid-19 Testing, Hospital Admission, and Intensive Care Among 2,026,227 United States Veterans Aged 54-75 Years. MedRxiv. 2020.

8. Khera R, Clark C, Lu Y, et al. Association of Angiotensin-Converting Enzyme Inhibitors and Angiotensin Receptor Blockers with the Risk of Hospitalization and Death in Hypertensive Patients with Coronavirus Disease-19. MedRxiv. 2020.

9. Hernan MA, Sauer BC, Hernandez-Diaz S, Platt R, Shrier I. Specifying a target trial prevents immortal time bias and other self-inflicted injuries in observational analyses. $J$ Clin Epidemiol. 2016;79:70-75.

10. Schneeweiss S. A basic study design for expedited safety signal evaluation based on electronic healthcare data. Pharmacoepidemiol Drug Saf. 2010;19(8):858-868.

11. Brookhart MA, Schneeweiss S, Rothman KJ, Glynn RJ, Avorn J, Stürmer T. Variable selection for propensity score models. Am J Epidemiol. 2006;163(12):1149-1156.

12. Rosenbaum PR, Rubin DB. The Central Role of the Propensity Score in Observational Studies for Causal Effects. Biometrika. 1983;70:41-55.

13. Ray WA. Evaluating medication effects outside of clinical trials: new-user designs. Am $J$ Epidemiol. 2003;158(9):915-920.

14. Rassen JA, Shelat AA, Myers J, Glynn RJ, Rothman KJ, Schneeweiss S. One-to-many propensity score matching in cohort studies. Pharmacoepidemiology and Drug Safety. 2012;21 Suppl 2:69-80.

15. Franklin JM, Rassen JA, Ackermann D, Bartels DB, Schneeweiss S. Metrics for covariate balance in cohort studies of causal effects. Stat Med. 2014;33(10):1685-1699.

16. Wang SV, Verpillat P, Rassen JA, Patrick A, Garry EM, Bartels DB. Transparency and Reproducibility of Observational Cohort Studies Using Large Healthcare Databases. Clin Pharmacol Ther. 2016;99(3):325-332. 
medRxiv preprint doi: https://doi.org/10.1101/2020.07.22.20159855; this version posted July 24, 2020. The copyright holder for this preprint

(which was not certified by peer review) is the author/funder, who has granted medRxiv a license to display the preprint in perpetuity.

It is made available under a CC-BY-NC-ND 4.0 International license .

17. Kim SC, Solomon DH, Rogers JR, et al. Cardiovascular Safety of Tocilizumab Versus Tumor Necrosis Factor Inhibitors in Patients With Rheumatoid Arthritis: A MultiDatabase Cohort Study. Arthritis Rheumatol. 2017;69(6):1154-1164.

18. Patorno E, Schneeweiss S, Gopalakrishnan C, Martin D, Franklin JM. Using Real-World Data to Predict Findings of an Ongoing Phase IV Cardiovascular Outcome Trial: Cardiovascular Safety of Linagliptin Versus Glimepiride. Diabetes Care. 2019;42(12):2204-2210.

19. Austin PC. Balance diagnostics for comparing the distribution of baseline covariates between treatment groups in propensity-score matched samples. Statistics in medicine. 2009;28(25):3083-3107.

20. Schneeweiss S, Robicsek A, Scranton R, Zuckerman D, Solomon DH. Veteran's affairs hospital discharge databases coded serious bacterial infections accurately. J Clin Epidemiol. 2007;60(4):397-409.

21. Johnson ES, Bartman BA, Briesacher BA, et al. The incident user design in comparative effectiveness research. Pharmacoepidemiology and Drug Safety. 2013;22(1):1-6.

22. Glynn RJ, Schneeweiss S, Sturmer T. Propensity scores in pharmacoepidemiology. Basic Clin Phar Tox. 2006;98:252-259.

23. Austin PC, Mamdani MM, Stukel TA, Anderson GM, Tu JV. The use of the propensity score for estimating treatment effects: administrative versus clinical data. Statistics in medicine. 2005;24(10):1563-1578.

24. Austin PC. Optimal caliper widths for propensity-score matching when estimating differences in means and differences in proportions in observational studies. Pharmaceutical statistics. 2011;10(2):150-161.

25. Suissa S, Garbe E. Primer: administrative health databases in observational studies of drug effects--advantages and disadvantages. Nat Clin Pract Rheumatol. 2007;3(12):725732. 


\section{Figure and Table Legend}

Figure 1: Cohort study design diagram

Table 1: Application of inclusion/exclusion criteria

Table 2: Baseline patient characteristics, comparing users of ARB vs. dhpCCB

Table 3: Baseline patient characteristics, comparing users of ACEi vs. dhpCCB

Table 4: 30-day risk and relative risk of hospitalization for COVID-19 infection among users of ARB vs. dhpCCB or ACEi vs. dhpCCB

\section{Appendix materials}

Appendix A: Coding algorithms for population and exposure

Appendix B: Coding algorithms for outcomes 
medRxiv preprint doi: https://doi.org/10.1101/2020.07.22.20159855; this version posted July 24, 2020. The copyright holder for this preprint (which was not certified by peer review) is the author/funder, who has granted medRxiv a license to display the preprint in perpetuity.

It is made available under a CC-BY-NC-ND 4.0 International license .

Figure 1: Cohort study design diagram



Abbreviations: ARB, angiotensin receptor blocker; ACEi, angiotensin converting enzyme inhibitor; dhpCCB, dihydropyridine calcium channel blocker; CKD, chronic kidney disease.

* Follow-up started the day after cohort entry until the earliest occurrence of a censoring event: outcome, 30 days after cohort entry, death, disenrollment, or end of study period (May 30, 2020). 
medRxiv preprint doi: https://doi.org/10.1101/2020.07.22.20159855; this version posted July 24, 2020. The copyright holder for this preprint

(which was not certified by peer review) is the author/funder, who has granted medRxiv a license to display the preprint in perpetuity.

It is made available under a CC-BY-NC-ND 4.0 International license .

Table 1: Application of inclusion/exclusion criteria

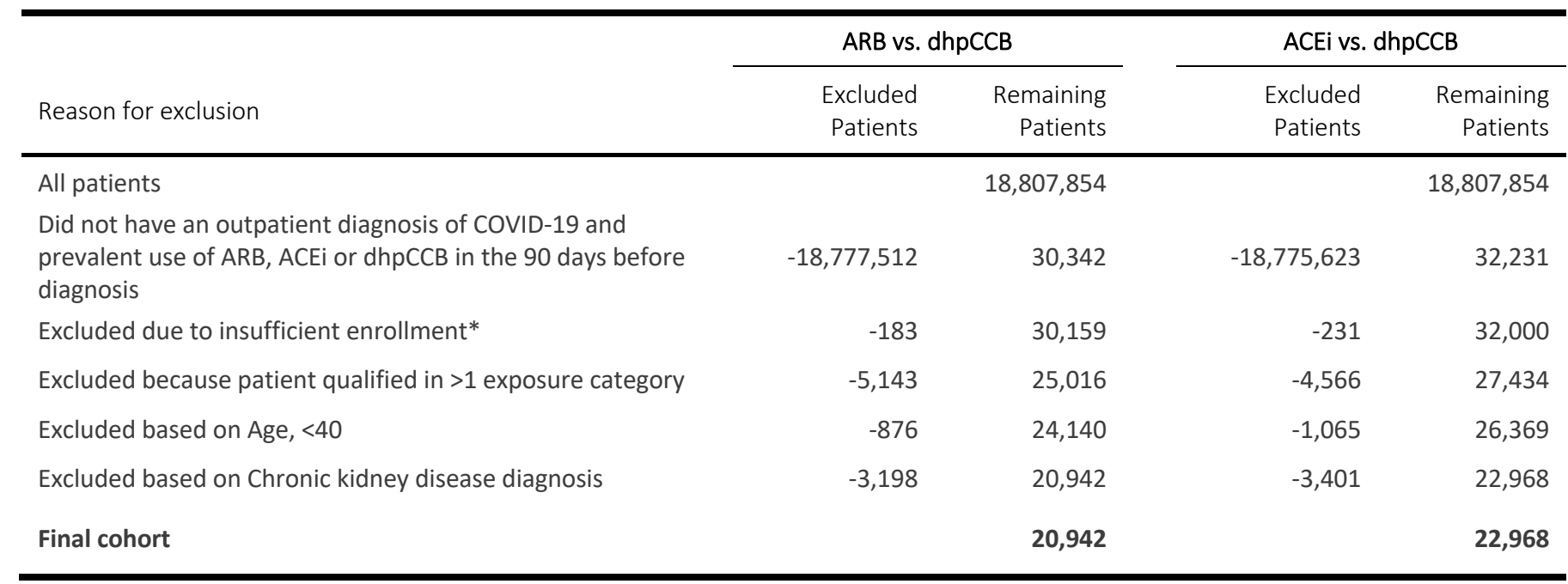

Abbreviations: ARB, angiotensin receptor blocker; ACEi, angiotensin converting enzyme inhibitor; dhpCCB, dihydropyridine calcium channel blocker.

* Insufficient enrollment was defined as less than 90 days of continuous enrollment. 
medRxiv preprint doi: https://doi.org/10.1101/2020.07.22.20159855; this version posted July 24, 2020. The copyright holder for this preprint (which was not certified by peer review) is the author/funder, who has granted medRxiv a license to display the preprint in perpetuity. It is made available under a CC-BY-NC-ND 4.0 International license .

Table 2: Baseline patient characteristics, comparing users of ARB vs. dhpCCB*

\begin{tabular}{|c|c|c|c|c|c|}
\hline \multirow[b]{2}{*}{ Variable } & \multicolumn{2}{|c|}{ Unmatched patients } & \multicolumn{3}{|c|}{ After 1:1 propensity-score matching } \\
\hline & ARB & dhpCCB & ARB & dhpCCB & $\begin{array}{l}\text { Standardized } \\
\text { difference }\end{array}$ \\
\hline Number of patients & 7,571 & 8,258 & 6,710 & 6,710 & \\
\hline Month/year of cohort entry date & & & & & 0.015 \\
\hline ...March 2020; n (\%) & $1,591(21.0 \%)$ & $1,441(17.4 \%)$ & $1,291(19.2 \%)$ & 1,315 (19.6\%) & \\
\hline ...April 2020; n (\%) & $5,029(66.4 \%)$ & $5,628(68.2 \%)$ & $4,535(67.6 \%)$ & 4,487 (66.9\%) & \\
\hline ...May 2020; n (\%) & 951 (12.6\%) & $1,189(14.4 \%)$ & $884(13.2 \%)$ & $908(13.5 \%)$ & \\
\hline Age; mean (sd) & $66.80(13.35)$ & $68.87(13.93)$ & $67.52(13.33)$ & $67.48(13.78)$ & 0.003 \\
\hline Female; n (\%) & $4,148(54.8 \%)$ & $4,283(51.9 \%)$ & $3,590(53.5 \%)$ & $3,596(53.6 \%)$ & 0.014 \\
\hline Alcohol abuse; n (\%) & $47(0.6 \%)$ & $114(1.4 \%)$ & $45(0.7 \%)$ & $45(0.7 \%)$ & 0.000 \\
\hline Coagulation deficiency; n (\%) & $78(1.0 \%)$ & $95(1.2 \%)$ & $66(1.0 \%)$ & $62(0.9 \%)$ & 0.006 \\
\hline Depression; n (\%) & $449(5.9 \%)$ & 707 (8.6\%) & $419(6.2 \%)$ & $396(5.9 \%)$ & 0.014 \\
\hline Drug abuse; n (\%) & $45(0.6 \%)$ & $103(1.2 \%)$ & $43(0.6 \%)$ & $48(0.7 \%)$ & 0.009 \\
\hline Fluid and electrolyte disorders; n (\%) & $23(0.3 \%)$ & $25(0.3 \%)$ & $19(0.3 \%)$ & $17(0.3 \%)$ & 0.006 \\
\hline HIV and AIDS; n (\%) & $24(0.3 \%)$ & $44(0.5 \%)$ & $24(0.4 \%)$ & $29(0.4 \%)$ & 0.012 \\
\hline Lymphoma; n (\%) & $29(0.4 \%)$ & $36(0.4 \%)$ & $29(0.4 \%)$ & $26(0.4 \%)$ & 0.007 \\
\hline Metastatic cancer; n (\%) & $44(0.6 \%)$ & $62(0.8 \%)$ & $41(0.6 \%)$ & $40(0.6 \%)$ & 0.002 \\
\hline Paralysis; n (\%) & $49(0.6 \%)$ & $112(1.4 \%)$ & $48(0.7 \%)$ & $51(0.8 \%)$ & 0.005 \\
\hline Psychoses; n (\%) & $103(1.4 \%)$ & $246(3.0 \%)$ & $103(1.5 \%)$ & $87(1.3 \%)$ & 0.020 \\
\hline Solid tumor without metastasis; n (\%) & $238(3.1 \%)$ & $278(3.4 \%)$ & $217(3.2 \%)$ & $216(3.2 \%)$ & 0.001 \\
\hline Valvular disease; n (\%) & $202(2.7 \%)$ & $164(2.0 \%)$ & $153(2.3 \%)$ & $136(2.0 \%)$ & 0.017 \\
\hline Weight loss; n (\%) & $80(1.1 \%)$ & $159(1.9 \%)$ & $78(1.2 \%)$ & $76(1.1 \%)$ & 0.003 \\
\hline Smoking; n (\%) & $186(2.5 \%)$ & $239(2.9 \%)$ & $172(2.6 \%)$ & $163(2.4 \%)$ & 0.009 \\
\hline Pregnancy; n (\%) & $4(0.1 \%)$ & $1(0.0 \%)$ & $2(0.0 \%)$ & $1(0.0 \%)$ & 0.010 \\
\hline Acute coronary syndrome; $\mathrm{n}(\%)$ & $96(1.3 \%)$ & $89(1.1 \%)$ & $80(1.2 \%)$ & $74(1.1 \%)$ & 0.008 \\
\hline Arrhythmias or cardiac dysrhythmias; n (\%) & $672(8.9 \%)$ & $683(8.3 \%)$ & $557(8.3 \%)$ & $532(7.9 \%)$ & 0.014 \\
\hline Asthma; n (\%) & $346(4.6 \%)$ & $303(3.7 \%)$ & $248(3.7 \%)$ & $251(3.7 \%)$ & 0.002 \\
\hline Cerebrovascular diseases; n (\%) & $342(4.5 \%)$ & $632(7.7 \%)$ & $324(4.8 \%)$ & $326(4.9 \%)$ & 0.001 \\
\hline Chronic kidney disease a; n (\%) & $0(0.0 \%)$ a & $0(0.0 \%)$ a & $0(0.0 \%)$ a & $0(0.0 \%)$ a & - \\
\hline Chronic pulmonary disease; $\mathrm{n}(\%)$ & $781(10.3 \%)$ & $897(10.9 \%)$ & $638(9.5 \%)$ & $645(9.6 \%)$ & 0.004 \\
\hline Cirrhosis; n (\%) & $25(0.3 \%)$ & $27(0.3 \%)$ & $19(0.3 \%)$ & $21(0.3 \%)$ & 0.005 \\
\hline Conduction disorders; $\mathrm{n}(\%)$ & $114(1.5 \%)$ & $120(1.5 \%)$ & $97(1.4 \%)$ & $83(1.2 \%)$ & 0.018 \\
\hline Congestive heart failure; n (\%) & $584(7.7 \%)$ & $508(6.2 \%)$ & $448(6.7 \%)$ & $428(6.4 \%)$ & 0.012 \\
\hline Cystic fibrosis; n (\%) & $0(0.0 \%)$ & $0(0.0 \%)$ & $0(0.0 \%)$ & $0(0.0 \%)$ & - \\
\hline Dependence on supplemental oxygen; n (\%) & $99(1.3 \%)$ & $136(1.6 \%)$ & $91(1.4 \%)$ & $92(1.4 \%)$ & 0.001 \\
\hline End-stage renal disease a; $\mathrm{n}(\%)$ & $0(0.0 \%) a$ & $0(0.0 \%) \mathrm{a}$ & $0(0.0 \%)$ a & $0(0.0 \%) \mathrm{a}$ & - \\
\hline Hematologic disorders; n (\%) & $92(1.2 \%)$ & $89(1.1 \%)$ & $73(1.1 \%)$ & $66(1.0 \%)$ & 0.010 \\
\hline Hyperlipidemia; n (\%) & $1,514(20.0 \%)$ & $1,522(18.4 \%)$ & $1,226(18.3 \%)$ & $1,202(17.9 \%)$ & 0.009 \\
\hline Hypertension; n (\%) & $2,702(35.7 \%)$ & $3,180(38.5 \%)$ & $2,329(34.7 \%)$ & $2,298(34.2 \%)$ & 0.010 \\
\hline $\begin{array}{l}\text { Inflammatory heart disorders (pericarditis, } \\
\text { endocarditis, myocarditis); n (\%) }\end{array}$ & 8 (0.1\%) & $10(0.1 \%)$ & 7 (0.1\%) & 5 (0.1\%) & 0.010 \\
\hline
\end{tabular}


medRxiv preprint doi: https://doi.org/10.1101/2020.07.22.20159855; this version posted July 24, 2020. The copyright holder for this preprint (which was not certified by peer review) is the author/funder, who has granted medRxiv a license to display the preprint in perpetuity.

It is made available under a CC-BY-NC-ND 4.0 International license .

\begin{tabular}{|c|c|c|c|c|c|}
\hline Ischemic heart disease; $\mathrm{n}(\%)$ & $691(9.1 \%)$ & $670(8.1 \%)$ & $564(8.4 \%)$ & $529(7.9 \%)$ & 0.019 \\
\hline Liver disease, mild; $\mathrm{n}(\%)$ & $119(1.6 \%)$ & $140(1.7 \%)$ & $103(1.5 \%)$ & $103(1.5 \%)$ & 0.000 \\
\hline Liver disease, moderate or severe; $\mathrm{n}(\%)$ & $9(0.1 \%)$ & $11(0.1 \%)$ & $7(0.1 \%)$ & $7(0.1 \%)$ & 0.000 \\
\hline Myocardial Infarction; n (\%) & $128(1.7 \%)$ & $130(1.6 \%)$ & $108(1.6 \%)$ & $106(1.6 \%)$ & 0.002 \\
\hline Overweight or obesity; $\mathrm{n}(\%)$ & $966(12.8 \%)$ & $830(10.1 \%)$ & $741(11.0 \%)$ & 735 (11.0\%) & 0.003 \\
\hline Obstructive sleep apnea; n (\%) & $351(4.6 \%)$ & $274(3.3 \%)$ & $258(3.8 \%)$ & $249(3.7 \%)$ & 0.007 \\
\hline Peptic ulcer disease; $\mathrm{n}(\%)$ & $24(0.3 \%)$ & $47(0.6 \%)$ & $23(0.3 \%)$ & $14(0.2 \%)$ & 0.026 \\
\hline Peripheral vascular disease; $\mathrm{n}(\%)$ & $386(5.1 \%)$ & $472(5.7 \%)$ & $337(5.0 \%)$ & $327(4.9 \%)$ & 0.007 \\
\hline Potassium or magnesium imbalance; $\mathrm{n}(\%)$ & $10(0.1 \%)$ & $9(0.1 \%)$ & $5(0.1 \%)$ & $7(0.1 \%)$ & 0.010 \\
\hline Psoriasis; n (\%) & $31(0.4 \%)$ & $21(0.3 \%)$ & $25(0.4 \%)$ & $17(0.3 \%)$ & 0.021 \\
\hline Pulmonary circulation disorders; $\mathrm{n}$ (\%) & $97(1.3 \%)$ & $113(1.4 \%)$ & $81(1.2 \%)$ & $82(1.2 \%)$ & 0.001 \\
\hline Renal impairment; $\mathrm{n}(\%)$ & $267(3.5 \%)$ & $418(5.1 \%)$ & $251(3.7 \%)$ & $256(3.8 \%)$ & 0.004 \\
\hline Rheumatic disease; $\mathrm{n}(\%)$ & $130(1.7 \%)$ & $122(1.5 \%)$ & $106(1.6 \%)$ & $101(1.5 \%)$ & 0.006 \\
\hline Stroke (ischemic, hemorrhagic); n (\%) & $148(2.0 \%)$ & $299(3.6 \%)$ & $144(2.1 \%)$ & $148(2.2 \%)$ & 0.004 \\
\hline Systemic lupus erythematosus; n (\%) & $14(0.2 \%)$ & $17(0.2 \%)$ & $12(0.2 \%)$ & $9(0.1 \%)$ & 0.011 \\
\hline Type-2 Diabetes; n (\%) & $1,562(20.6 \%)$ & $1,608(19.5 \%)$ & $1,272(19.0 \%)$ & $1,251(18.6 \%)$ & 0.008 \\
\hline All cause pneumonia; $n$ (\%) & $651(8.6 \%)$ & $834(10.1 \%)$ & $598(8.9 \%)$ & $589(8.8 \%)$ & 0.005 \\
\hline Use of any anticoagulant; $n(\%)$ & $797(10.5 \%)$ & $808(9.8 \%)$ & $657(9.8 \%)$ & $664(9.9 \%)$ & 0.004 \\
\hline Use of any novel oral anticoagulant; $n(\%)$ & $631(8.3 \%)$ & $622(7.5 \%)$ & $529(7.9 \%)$ & $533(7.9 \%)$ & 0.002 \\
\hline Use of warfarin; $n(\%)$ & $154(2.0 \%)$ & $133(1.6 \%)$ & $113(1.7 \%)$ & $118(1.8 \%)$ & 0.006 \\
\hline Use of any anti-platelet medication; n (\%) & $1,075(14.2 \%)$ & $1,356(16.4 \%)$ & $998(14.9 \%)$ & $975(14.5 \%)$ & 0.010 \\
\hline Use of corticosteroid, inhaled; $n(\%)$ & $749(9.9 \%)$ & $691(8.4 \%)$ & $589(8.8 \%)$ & $588(8.8 \%)$ & 0.001 \\
\hline Use of any asthma medication; $\mathrm{n}(\%)$ & $2,372(31.3 \%)$ & $2,374(28.7 \%)$ & $2,002(29.8 \%)$ & $1,999(29.8 \%)$ & 0.001 \\
\hline Any inpatient medical claim; n (\%) & $1,452(19.2 \%)$ & $2,148(26.0 \%)$ & $1,354(20.2 \%)$ & $1,305(19.4 \%)$ & 0.018 \\
\hline Coronary care unit visit; n (\%) & $20(0.3 \%)$ & $26(0.3 \%)$ & $20(0.3 \%)$ & $23(0.3 \%)$ & 0.008 \\
\hline Intensive care unit revenue codes; n (\%) & $64(0.8 \%)$ & $68(0.8 \%)$ & $50(0.7 \%)$ & $48(0.7 \%)$ & 0.004 \\
\hline No. of any physician visit; mean (sd) & $1.18(2.50)$ & $0.92(2.46)$ & $1.04(2.14)$ & $1.01(2.65)$ & 0.010 \\
\hline No. of pharmacy dispensings; mean (sd) & $6.23(6.18)$ & $6.61(6.86)$ & $6.25(6.27)$ & $6.18(6.39)$ & 0.011 \\
\hline
\end{tabular}

Abbreviations: ARB, angiotensin receptor blocker; ACEi, angiotensin converting enzyme inhibitor; dhpCCB, dihydropyridine calcium channel blocker; sd, standard deviation; No., number.

* Patients were ongoing users of either ARB or dhpCCB in the 90 days before cohort entry (documented COVID-19 infection); all patients characteristics were assessed during the 90 days before cohort entry.

a The baseline prevalence of chronic kidney disease and end stage renal disease was $0 \%$ as it was a study exclusion criterion. 
medRxiv preprint doi: https://doi.org/10.1101/2020.07.22.20159855; this version posted July 24, 2020. The copyright holder for this preprint (which was not certified by peer review) is the author/funder, who has granted medRxiv a license to display the preprint in perpetuity. It is made available under a CC-BY-NC-ND 4.0 International license .

Table 3: Baseline patient characteristics, comparing users of ACEi vs. dhpCCB*

\begin{tabular}{|c|c|c|c|c|c|}
\hline \multirow[b]{2}{*}{ Variable } & \multicolumn{2}{|c|}{ Unmatched patients } & \multicolumn{3}{|c|}{ After 1:1 propensity-score matching } \\
\hline & ACEi & dhpCCB & ACEi & dhpCCB & $\begin{array}{l}\text { Standardized } \\
\text { difference }\end{array}$ \\
\hline Number of patients & 8,484 & 8,653 & 7,334 & 7,334 & \\
\hline Month/year of cohort entry date & & & & & 0.009 \\
\hline ...March 2020; n (\%) & $1,527(18.0 \%)$ & $1,592(18.4 \%)$ & $1,306(17.8 \%)$ & $1,330(18.1 \%)$ & \\
\hline ...April 2020; n (\%) & $5,712(67.3 \%)$ & $5,876(67.9 \%)$ & $4,976(67.8 \%)$ & 4,965 (67.7\%) & \\
\hline ...May 2020; n (\%) & 1,245 (14.7\%) & $1,185(13.7 \%)$ & $1,052(14.3 \%)$ & $1,039(14.2 \%)$ & \\
\hline Age; mean (sd) & $66.06(13.19)$ & $68.90(13.91)$ & $66.97(13.17)$ & $67.13(13.48)$ & 0.012 \\
\hline Female; n (\%) & $3,990(47.0 \%)$ & $4,706(54.4 \%)$ & $3,695(50.4 \%)$ & $3,680(50.2 \%)$ & 0.004 \\
\hline Alcohol abuse; n (\%) & 109 (1.3\%) & $106(1.2 \%)$ & $93(1.3 \%)$ & $87(1.2 \%)$ & 0.007 \\
\hline Coagulation deficiency; $\mathrm{n}(\%)$ & $102(1.2 \%)$ & $88(1.0 \%)$ & $67(0.9 \%)$ & 75 (1.0\%) & 0.011 \\
\hline Depression; n (\%) & $649(7.6 \%)$ & $645(7.5 \%)$ & $530(7.2 \%)$ & $506(6.9 \%)$ & 0.013 \\
\hline Drug abuse; n (\%) & $104(1.2 \%)$ & $87(1.0 \%)$ & $87(1.2 \%)$ & $79(1.1 \%)$ & 0.010 \\
\hline Fluid and electrolyte disorders; $n$ (\%) & $22(0.3 \%)$ & $17(0.2 \%)$ & $14(0.2 \%)$ & $14(0.2 \%)$ & 0.000 \\
\hline HIV and AIDS; n (\%) & $47(0.6 \%)$ & $37(0.4 \%)$ & $37(0.5 \%)$ & $36(0.5 \%)$ & 0.002 \\
\hline Lymphoma; n (\%) & $31(0.4 \%)$ & $47(0.5 \%)$ & $27(0.4 \%)$ & $27(0.4 \%)$ & 0.000 \\
\hline Metastatic cancer; n (\%) & $42(0.5 \%)$ & $66(0.8 \%)$ & $39(0.5 \%)$ & $32(0.4 \%)$ & 0.014 \\
\hline Paralysis; n (\%) & $85(1.0 \%)$ & $105(1.2 \%)$ & $76(1.0 \%)$ & $83(1.1 \%)$ & 0.009 \\
\hline Psychoses; n (\%) & $222(2.6 \%)$ & $195(2.3 \%)$ & $166(2.3 \%)$ & $167(2.3 \%)$ & 0.001 \\
\hline Solid tumor without metastasis; n (\%) & $257(3.0 \%)$ & $287(3.3 \%)$ & $221(3.0 \%)$ & $211(2.9 \%)$ & 0.008 \\
\hline Valvular disease; n (\%) & $213(2.5 \%)$ & $186(2.1 \%)$ & $145(2.0 \%)$ & $156(2.1 \%)$ & 0.011 \\
\hline Weight loss; n (\%) & $121(1.4 \%)$ & $152(1.8 \%)$ & $106(1.4 \%)$ & $100(1.4 \%)$ & 0.007 \\
\hline Smoking; $\mathrm{n}(\%)$ & $275(3.2 \%)$ & $246(2.8 \%)$ & $202(2.8 \%)$ & $208(2.8 \%)$ & 0.005 \\
\hline Pregnancy; n (\%) & $1(0.0 \%)$ & $0(0.0 \%)$ & $0(0.0 \%)$ & $0(0.0 \%)$ & - \\
\hline Acute coronary syndrome; $\mathrm{n}(\%)$ & $108(1.3 \%)$ & $88(1.0 \%)$ & $77(1.0 \%)$ & $76(1.0 \%)$ & 0.001 \\
\hline Arrhythmias or cardiac dysrhythmias; $n$ (\%) & $803(9.5 \%)$ & $693(8.0 \%)$ & $581(7.9 \%)$ & $593(8.1 \%)$ & 0.006 \\
\hline Asthma; n (\%) & $308(3.6 \%)$ & $339(3.9 \%)$ & $267(3.6 \%)$ & $265(3.6 \%)$ & 0.001 \\
\hline Cerebrovascular diseases; $n$ (\%) & $482(5.7 \%)$ & $589(6.8 \%)$ & $434(5.9 \%)$ & $416(5.7 \%)$ & 0.011 \\
\hline Chronic kidney disease a; $\mathrm{n}(\%)$ & $0(0.0 \%)$ a & $0(0.0 \%)$ a & $0(0.0 \%)$ a & $0(0.0 \%)$ a & - \\
\hline Chronic pulmonary disease; $\mathrm{n}(\%)$ & $884(10.4 \%)$ & $928(10.7 \%)$ & $748(10.2 \%)$ & $750(10.2 \%)$ & 0.001 \\
\hline Cirrhosis; n (\%) & $43(0.5 \%)$ & $32(0.4 \%)$ & $31(0.4 \%)$ & $31(0.4 \%)$ & 0.000 \\
\hline Conduction disorders; $\mathrm{n}(\%)$ & $117(1.4 \%)$ & $118(1.4 \%)$ & $88(1.2 \%)$ & $86(1.2 \%)$ & 0.003 \\
\hline Congestive heart failure; $\mathrm{n}(\%)$ & $655(7.7 \%)$ & $523(6.0 \%)$ & $452(6.2 \%)$ & $464(6.3 \%)$ & 0.007 \\
\hline Cystic fibrosis; n (\%) & $0(0.0 \%)$ & $0(0.0 \%)$ & $0(0.0 \%)$ & $0(0.0 \%)$ & - \\
\hline Dependence on supplemental oxygen; $\mathrm{n}(\%)$ & $132(1.6 \%)$ & $150(1.7 \%)$ & $111(1.5 \%)$ & $111(1.5 \%)$ & 0.000 \\
\hline End-stage renal disease a; $\mathrm{n}(\%)$ & $0(0.0 \%)$ a & $0(0.0 \%)$ a & $0(0.0 \%) \mathrm{a}$ & $0(0.0 \%)$ a & - \\
\hline Hematologic disorders; n (\%) & $119(1.4 \%)$ & $103(1.2 \%)$ & $80(1.1 \%)$ & $90(1.2 \%)$ & 0.013 \\
\hline Hyperlipidemia; n (\%) & $1,732(20.4 \%)$ & $1,595(18.4 \%)$ & $1,356(18.5 \%)$ & $1,378(18.8 \%)$ & 0.008 \\
\hline Hypertension; n (\%) & $3,003(35.4 \%)$ & 3,277 (37.9\%) & $2,561(34.9 \%)$ & $2,575(35.1 \%)$ & 0.004 \\
\hline $\begin{array}{l}\text { Inflammatory heart disorders (pericarditis, } \\
\text { endocarditis, myocarditis); } \mathrm{n}(\%)\end{array}$ & $7(0.1 \%)$ & $10(0.1 \%)$ & $7(0.1 \%)$ & $8(0.1 \%)$ & 0.004 \\
\hline
\end{tabular}


medRxiv preprint doi: https://doi.org/10.1101/2020.07.22.20159855; this version posted July 24, 2020. The copyright holder for this preprint (which was not certified by peer review) is the author/funder, who has granted medRxiv a license to display the preprint in perpetuity.

It is made available under a CC-BY-NC-ND 4.0 International license .

\begin{tabular}{|c|c|c|c|c|c|}
\hline Ischemic heart disease; $\mathrm{n}(\%)$ & 760 (9.0\%) & $679(7.8 \%)$ & $560(7.6 \%)$ & $573(7.8 \%)$ & 0.007 \\
\hline Liver disease, mild; n (\%) & $171(2.0 \%)$ & $142(1.6 \%)$ & $131(1.8 \%)$ & $121(1.6 \%)$ & 0.010 \\
\hline Liver disease, moderate or severe; $\mathrm{n}(\%)$ & $18(0.2 \%)$ & $12(0.1 \%)$ & $14(0.2 \%)$ & $12(0.2 \%)$ & 0.006 \\
\hline Myocardial Infarction; n (\%) & $171(2.0 \%)$ & $121(1.4 \%)$ & $106(1.4 \%)$ & $109(1.5 \%)$ & 0.003 \\
\hline Overweight or obesity; $\mathrm{n}(\%)$ & $1,022(12.0 \%)$ & $912(10.5 \%)$ & 794 (10.8\%) & $810(11.0 \%)$ & 0.007 \\
\hline Obstructive sleep apnea; n (\%) & $323(3.8 \%)$ & $344(4.0 \%)$ & $273(3.7 \%)$ & $295(4.0 \%)$ & 0.016 \\
\hline Peptic ulcer disease; n (\%) & $24(0.3 \%)$ & $40(0.5 \%)$ & $22(0.3 \%)$ & $23(0.3 \%)$ & 0.002 \\
\hline Peripheral vascular disease; $\mathrm{n}(\%)$ & $479(5.6 \%)$ & $446(5.2 \%)$ & $365(5.0 \%)$ & $370(5.0 \%)$ & 0.003 \\
\hline Potassium or magnesium imbalance; $\mathrm{n}(\%)$ & $3(0.0 \%)$ & $7(0.1 \%)$ & $3(0.0 \%)$ & $3(0.0 \%)$ & 0.000 \\
\hline Psoriasis; n (\%) & $21(0.2 \%)$ & $25(0.3 \%)$ & $21(0.3 \%)$ & $19(0.3 \%)$ & 0.005 \\
\hline Pulmonary circulation disorders; $\mathrm{n}$ (\%) & $102(1.2 \%)$ & $123(1.4 \%)$ & $84(1.1 \%)$ & $84(1.1 \%)$ & 0.000 \\
\hline Renal impairment; $\mathrm{n}(\%)$ & $308(3.6 \%)$ & $417(4.8 \%)$ & $285(3.9 \%)$ & $283(3.9 \%)$ & 0.001 \\
\hline Rheumatic disease; $\mathrm{n}(\%)$ & $93(1.1 \%)$ & $141(1.6 \%)$ & $82(1.1 \%)$ & $89(1.2 \%)$ & 0.009 \\
\hline Stroke (ischemic, hemorrhagic); n (\%) & $228(2.7 \%)$ & $278(3.2 \%)$ & $207(2.8 \%)$ & $207(2.8 \%)$ & 0.000 \\
\hline Systemic lupus erythematosus; $n$ (\%) & $20(0.2 \%)$ & $25(0.3 \%)$ & $15(0.2 \%)$ & $19(0.3 \%)$ & 0.011 \\
\hline Type-2 Diabetes; n (\%) & $2,029(23.9 \%)$ & $1,603(18.5 \%)$ & $1,446(19.7 \%)$ & $1,486(20.3 \%)$ & 0.014 \\
\hline All cause pneumonia; $n(\%)$ & $745(8.8 \%)$ & $869(10.0 \%)$ & $650(8.9 \%)$ & $668(9.1 \%)$ & 0.009 \\
\hline Use of any anticoagulant; $n(\%)$ & $885(10.4 \%)$ & $813(9.4 \%)$ & $688(9.4 \%)$ & $697(9.5 \%)$ & 0.004 \\
\hline Use of any novel oral anticoagulant; n (\%) & $645(7.6 \%)$ & $614(7.1 \%)$ & $524(7.1 \%)$ & $534(7.3 \%)$ & 0.005 \\
\hline Use of warfarin; $n(\%)$ & $217(2.6 \%)$ & $147(1.7 \%)$ & $134(1.8 \%)$ & $139(1.9 \%)$ & 0.005 \\
\hline Use of any anti-platelet medication; $\mathrm{n}(\%)$ & $1,259(14.8 \%)$ & $1,406(16.2 \%)$ & $1,100(15.0 \%)$ & $1,103(15.0 \%)$ & 0.001 \\
\hline Use of corticosteroid, inhaled; $n(\%)$ & $667(7.9 \%)$ & $786(9.1 \%)$ & $595(8.1 \%)$ & $638(8.7 \%)$ & 0.021 \\
\hline Use of any asthma medication; $n$ (\%) & $2,260(26.6 \%)$ & $2,636(30.5 \%)$ & $2,037(27.8 \%)$ & $2,096(28.6 \%)$ & 0.018 \\
\hline Any inpatient medical claim; n (\%) & $1,879(22.1 \%)$ & $2,068(23.9 \%)$ & $1,593(21.7 \%)$ & $1,613(22.0 \%)$ & 0.007 \\
\hline Coronary care unit visit; n (\%) & $25(0.3 \%)$ & $21(0.2 \%)$ & $19(0.3 \%)$ & $15(0.2 \%)$ & 0.011 \\
\hline Intensive care unit revenue codes; n (\%) & $67(0.8 \%)$ & $65(0.8 \%)$ & $49(0.7 \%)$ & $50(0.7 \%)$ & 0.002 \\
\hline No. of any physician visit; mean (sd) & $1.06(2.49)$ & $0.95(2.30)$ & $0.94(2.12)$ & $0.96(2.32)$ & 0.007 \\
\hline No. of pharmacy dispensings; mean (sd) & $6.17(6.31)$ & $6.54(6.66)$ & $6.18(6.36)$ & $6.22(6.44)$ & 0.007 \\
\hline
\end{tabular}

Abbreviations: ARB, angiotensin receptor blocker; ACEi, angiotensin converting enzyme inhibitor; dhpCCB, dihydropyridine calcium channel blocker; sd, standard deviation; No., number.

* Patients were ongoing users of either ACEi or dhpCCB in the 90 days before cohort entry (documented COVID-19 infection); all patients characteristics were assessed during the 90 days before cohort entry.

a The baseline prevalence of chronic kidney disease and end stage renal disease was $0 \%$ as it was a study exclusion criterion. 
Table 4: 30-day risk and relative risk of hospitalization for COVID-19 infection among users of ARB vs. dhpCCB or ACEi vs. dhpCCB

\begin{tabular}{|c|c|c|c|c|c|c|c|c|c|c|c|}
\hline \multirow[b]{2}{*}{ Outcome } & \multirow[b]{2}{*}{ Comparison } & \multicolumn{5}{|c|}{ Unmatched patients } & \multicolumn{5}{|c|}{ 1:1 propensity score matched patients } \\
\hline & & $\begin{array}{l}\mathrm{N} \text { of } \\
\text { patients }\end{array}$ & $\begin{array}{l}\mathrm{N} \text { of } \\
\text { events }\end{array}$ & $\begin{array}{l}\text { 30-day } \\
\text { risk }\end{array}$ & Risk Ratio (95\% Cl) & Risk difference $(95 \% \mathrm{Cl})$ & $\begin{array}{l}\mathrm{N} \text { of } \\
\text { patients }\end{array}$ & $\begin{array}{l}\mathrm{N} \text { of } \\
\text { events }\end{array}$ & $\begin{array}{l}\text { 30-day } \\
\text { risk }\end{array}$ & Risk Ratio (95\% Cl) & Risk difference $(95 \% \mathrm{Cl})$ \\
\hline \multirow{4}{*}{$\begin{array}{l}\text { Hospitalization } \\
\text { for COVID-19 }\end{array}$} & ARB & 7,571 & 201 & $2.66 \%$ & $0.72(0.61,0.86)$ & $-1.02 \%(-1.58,-0.47)$ & 6,710 & 191 & $2.85 \%$ & $0.83(0.68,1.00)$ & $-0.59 \%(-1.20,0.01)$ \\
\hline & dhpCCB & 8,258 & 304 & $3.68 \%$ & referent & referent & 6,710 & 231 & $3.44 \%$ & referent & referent \\
\hline & ACEi & 8,484 & 246 & $2.90 \%$ & $0.79(0.67,0.93)$ & $-0.76 \%(-1.31,-0.22)$ & 7,334 & 229 & $3.12 \%$ & $0.90(0.76,1.07)$ & $-0.34 \%(-0.93,0.25)$ \\
\hline & dhpCCB & 8,653 & 317 & $3.66 \%$ & referent & referent & 7,334 & 254 & $3.46 \%$ & referent & referent \\
\hline \multirow{4}{*}{$\begin{array}{l}\text { Hospitalization with } \\
\text { acute organ } \\
\text { dysfunction }^{b}\end{array}$} & ARB & 7,571 & 85 & $1.12 \%$ & $0.81(0.62,1.08)$ & $-0.26 \%(-0.62,0.10)$ & 6,710 & 76 & $1.13 \%$ & $0.88(0.65,1.20)$ & $-0.15 \%(-0.53,0.24)$ \\
\hline & dhpCCB & 8,258 & 114 & $1.38 \%$ & referent & referent & 6,710 & 86 & $1.28 \%$ & referent & referent \\
\hline & ACEi & 8,484 & 104 & $1.23 \%$ & $0.94(0.72,1.22)$ & $-0.08 \%(-0.43,0.27)$ & 7,334 & 97 & $1.32 \%$ & $1.00(0.76,1.32)$ & $0.00(-0.37,0.37)$ \\
\hline & dhpCCB & 8,653 & 113 & $1.31 \%$ & referent & referent & 7,334 & 97 & $1.32 \%$ & referent & referent \\
\hline \multirow{4}{*}{$\begin{array}{l}\text { Hospitalization with } \\
\text { acute respiratory } \\
\text { distress syndrome }\end{array}$} & ARB & 7,571 & 87 & $1.15 \%$ & $0.78(0.60,1.03)$ & $-0.32 \%(-0.68,0.05)$ & 6,710 & 78 & $1.16 \%$ & $0.86(0.63,1.16)$ & $-0.20 \%(-0.59,0.20)$ \\
\hline & dhpCCB & 8,258 & 121 & $1.47 \%$ & referent & referent & 6,710 & 91 & $1.36 \%$ & referent & referent \\
\hline & ACEi & 8,484 & 102 & $1.20 \%$ & $0.90(0.69,1.18)$ & $-0.13 \%(-0.47,0.22)$ & 7,334 & 97 & $1.32 \%$ & $0.99(0.75,1.31)$ & $-0.01 \%(-0.40,0.37)$ \\
\hline & dhpCCB & 8,653 & 115 & $1.33 \%$ & referent & referent & 7,334 & 98 & $1.33 \%$ & referent & referent \\
\hline \multirow{4}{*}{$\begin{array}{l}\text { Hospitalization with } \\
\text { sepsis }\end{array}$} & ARB & 7,571 & 54 & $0.71 \%$ & $0.69(0.49,0.97)$ & $-0.32 \%(-0.62,-0.02)$ & 6,710 & 46 & $0.69 \%$ & $0.71(0.49,1.03)$ & $-0.28 \%(-0.60,0.04)$ \\
\hline & dhpCCB & 8,258 & 85 & $1.03 \%$ & referent & referent & 6,710 & 65 & $0.97 \%$ & referent & referent \\
\hline & ACEi & 8,484 & 76 & $0.89 \%$ & $0.92(0.68,1.26)$ & $-0.08 \%(-0.38,0.23)$ & 7,334 & 71 & $0.97 \%$ & $1.06(0.76,1.48)$ & $0.06 \%(-0.27,0.38)$ \\
\hline & dhpCCB & 8,653 & 84 & $0.97 \%$ & referent & referent & 7,334 & 67 & $0.91 \%$ & referent & referent \\
\hline \multirow{4}{*}{$\begin{array}{l}\text { Hospitalization with } \\
\text { respiratory intubation } \\
\text { or mechanical } \\
\text { ventilation }\end{array}$} & ARB & 7,571 & 113 & $1.49 \%$ & $0.87(0.68,1.11)$ & $-0.23 \%(-0.63,0.18)$ & 6,710 & 92 & $1.37 \%$ & $0.77(0.59,1.00)$ & $-0.42 \%(-0.85,0.02)$ \\
\hline & dhpCCB & 8,258 & 142 & $1.72 \%$ & referent & referent & 6,710 & 120 & $1.79 \%$ & referent & referent \\
\hline & ACEi & 8,484 & 158 & $1.86 \%$ & $0.99(0.80,1.23)$ & $-0.02 \%(-0.44,0.39)$ & 7,334 & 131 & $1.79 \%$ & $0.99(0.78,1.26)$ & $-0.01 \%(-0.46,0.43)$ \\
\hline & dhpCCB & 8,653 & 163 & $1.88 \%$ & referent & referent & 7,334 & 132 & $1.80 \%$ & referent & referent \\
\hline
\end{tabular}

Abbreviations: ARB, angiotensin receptor blocker; ACEi, angiotensin converting enzyme inhibitor; dhpCCB, dihydropyridine calcium channel blocker; N, number; Pts, patients; 95\% CI, 95\% confidence interval.

${ }^{a}$ The baseline prevalence of chronic kidney disease and end stage renal disease was $0 \%$ as it was a study exclusion criterion; ${ }^{\mathrm{b}}$ Acute organ dysfunction includes: Critical illness polyneuropathy, Acute kidney failure unspecified, Metabolic encephalopathy, Acute respiratory failure, Acute respiratory failure with hypoxia, Acute and subacute hepatic failure with coma, Acute kidney failure, Acute kidney failure with acute cortical necrosis, Disseminated intravascular coagulation, Acute and subacute hepatic failure, Acute and subacute hepatic failure without coma, Acute kidney failure with medullary necrosis, Acute respiratory failure, unspecified whether with hypoxia or hypercapnia, Acute respiratory failure with hypercapnia, Other acute kidney failure, Critical illness myopathy, Acute kidney failure with tubular necrosis 
medRxiv preprint doi: https://doi.org/10.1101/2020.07.22.20159855; this version posted July 24, 2020. The copyright holder for this preprint (which was not certified by peer review) is the author/funder, who has granted medRxiv a license to display the preprint in perpetuity.

It is made available under a CC-BY-NC-ND 4.0 International license .

\section{Appendix A: Coding algorithms for population and exposure}

REFERENT: dhpCCB (Excludes: verapamil and diltiazem).

- The occurrence of Pharmacy Claims with the following attributes:

- Generic Name is any of: $\{$ "CLEVIDIPINE BUTYRATE", "ALISKIREN

HEMIFUMARATE/AMLODIPINE/HYDROCHLOROTHIAZIDE", "MIBEFRADIL DI-HCL", "ALISKIREN HEMIFUMARATE/AMLODIPINE BESYLATE", "BEPRIDIL HCL", "NICARDIPINE IN DEXTROSE, ISOOSMOTIC", "NICARDIPINE IN SODIUM CHLORIDE, ISO-OSMOTIC", "NIMODIPINE", "ISRADIPINE", "NICARDIPINE HCL", "AMLODIPINE BESYLATE/ATORVASTATIN CALCIUM", "FELODIPINE", "AMLODIPINE BESYLATE", "NIFEDIPINE" \}

- CLEVIDIPINE BUTYRATE

- MIBEFRADIL DI-HCL

- bEPRIDIL HCL

- nicARDipine in DEXTROSE, ISO-OSMOTIC

- NICARDIPINE IN SODIUM CHLORIDE, ISO-OSMOTIC

- NIMODIPINE

- isRadipINE

- NICARDIPINE HCL

- AMLODIPINE BESYLATE/ATORVASTATIN CALCIUM

- FELODIPINE

- AMLODIPINE BESYLATE

- NIFEDIPINE

EXPOSURE: ACEi

- The occurrence of Pharmacy Claims with the following attributes:

- Generic Name is any of: \{ "AMLODIPINE BESYLATE/BENAZEPRIL HCL", "BENAZEPRIL HCL", "BENAZEPRIL HCL/HYDROCHLOROTHIAZIDE", "CAPTOPRIL", "CAPTOPRIL/HYDROCHLOROTHIAZIDE", "ENALAPRIL MALEATE", "ENALAPRIL MALEATE/DILTIAZEM MALATE", "ENALAPRIL MALEATE/FELODIPINE", "ENALAPRIL MALEATE/HYDROCHLOROTHIAZIDE", "ENALAPRILAT DIHYDRATE", "FOSINOPRIL SODIUM", "FOSINOPRIL SODIUM/HYDROCHLOROTHIAZIDE", "LISINOPRIL", "LISINOPRIL/DIETARY SUPPLEMENT,COMB.10", "LISINOPRIL/HYDROCHLOROTHIAZIDE”, "MOEXIPRIL HCL", "MOEXIPRIL HCL/HYDROCHLOROTHIAZIDE", "PERINDOPRIL ARGININE/AMLODIPINE BESYLATE", "PERINDOPRIL ERBUMINE", "QUINAPRIL HCL", “QUINAPRIL HCL/HYDROCHLOROTHIAZIDE", "RAMIPRIL", "TRANDOLAPRIL", "TRANDOLAPRIL/VERAPAMIL $\left.\mathrm{HCL}^{\prime \prime}\right\}$

- BENAZEPRIL HCL

- BENAZEPRIL HCL/HYDROCHLOROTHIAZIDE

- CAPTOPRIL

- CAPTOPRIL/HYDROCHLOROTHIAZIDE

- enAlapril maleate

- ENALAPRIL MALEATE/HYDROCHLOROTHIAZIDE

- eNALAPRILAT DIHYDRATE

- FOSINOPRIL SODIUM

- FOSINOPRIL SODIUM/HYDROCHLOROTHIAZIDE

- LISINOPRIL 
medRxiv preprint doi: https://doi.org/10.1101/2020.07.22.20159855; this version posted July 24, 2020. The copyright holder for this preprint (which was not certified by peer review) is the author/funder, who has granted medRxiv a license to display the preprint in perpetuity. It is made available under a CC-BY-NC-ND 4.0 International license .

- LISINOPRIL/DIETARY SUPPLEMENT,COMB.10

- $\quad$ LISINOPRIL/HYDROCHLOROTHIAZIDE

- MOEXIPRIL HCL

- MOEXIPRIL HCL/HYDROCHLOROTHIAZIDE

- PERINDOPRIL ERBUMINE

- QUINAPRIL HCL

- QUINAPRIL HCL/HYDROCHLOROTHIAZIDE

- RAMIPRIL

- tRANDOLAPRIL

- The occurrence of Pharmacy Claims with the following attributes:

- Brand Name is any of: $\{$ "ACCUPRIL", "ACEON", "ALTACE", "CAPOTEN", "LOTENSIN", "LOTENSIN HCT", "MAVIK", "MONOPRIL", "MONOPRIL HCT", "PRINIVIL", "UNIVASC", "VASOTEC", "VASOTEC I.V.", "ZESTRIL" \}

- ACCUPRIL

- ACEON

- ALTACE

- cAPOTEN

- LOTENSIN

- LOTENSIN HCT

- MAVIK

- MONOPRIL

- MONOPRIL HCT

- PRINIVIL

- UNIVASC

- VASOTEC

- VASOTECI.V.

- ZESTRIL

EXPOSURE: ARB

- The occurrence of Pharmacy Claims with the following attributes:

- Generic Name is any of: \{ "ALISKIREN/VALSARTAN", "AMLODIPINE BESYLATE/OLMESARTAN MEDOXOMIL", "AMLODIPINE BESYLATE/VALSARTAN", "AMLODIPINE BESYLATE/VALSARTAN/HYDROCHLOROTHIAZIDE", "AZILSARTAN MEDOXOMIL", "AZILSARTAN MEDOXOMIL/CHLORTHALIDONE", "CANDESARTAN CILEXETIL", "CANDESARTAN CILEXETIL/HYDROCHLOROTHIAZIDE", "EPROSARTAN MESYLATE", "EPROSARTAN MESYLATE/HYDROCHLOROTHIAZIDE", "IRBESARTAN", "IRBESARTAN/HYDROCHLOROTHIAZIDE", "LOSARTAN POTASSIUM", "LOSARTAN POTASSIUM/HYDROCHLOROTHIAZIDE", "NEBIVOLOL HCL/VALSARTAN", "OLMESARTAN MEDOXOMIL", "OLMESARTAN MEDOXOMIL/AMLODIPINE BESYLATE/HYDROCHLOROTHIAZIDE", "OLMESARTAN MEDOXOMIL/HYDROCHLOROTHIAZIDE", "SACUBITRIL/VALSARTAN", "TELMISARTAN", "TELMISARTAN/AMLODIPINE BESYLATE", "TELMISARTAN/HYDROCHLOROTHIAZIDE", "VALSARTAN", "VALSARTAN/HYDROCHLOROTHIAZIDE" \}

- ALISKIREN/VALSARTAN

- AZILSARTAN MEDOXOMIL

- aZILSARTAN MEDOXOMIL/CHLORTHALIDONE

- candeSARTAN CILEXETIL 
medRxiv preprint doi: https://doi.org/10.1101/2020.07.22.20159855; this version posted July 24, 2020. The copyright holder for this preprint (which was not certified by peer review) is the author/funder, who has granted medRxiv a license to display the preprint in perpetuity. It is made available under a CC-BY-NC-ND 4.0 International license .

- CANDESARTAN CILEXETIL/HYDROCHLOROTHIAZIDE

- eprosartan mesylate

- ePROSARTAN MESYLATE/HYDROCHLOROTHIAZIDE

- IRBESARTAN

- IRBESARTAN/HYDROCHLOROTHIAZIDE

- losartan POTASSIUM

- lOSARTAN POTASSIUM/HYDROCHLOROTHIAZIDE

- NEBIVOLOL HCL/VALSARTAN

- OLMESARTAN MEDOXOMIL

- OLMESARTAN MEDOXOMIL/HYDROCHLOROTHIAZIDE

- sacubitril/VALSARTAN

- telmisartan

- telMISARTAN/HYDROCHLOROTHIAZIDE

- VALSARTAN

- VALSARTAN/HYDROCHLOROTHIAZIDE

- The occurrence of Pharmacy Claims with the following attributes:

- Brand Name is any of: $\{$ "ATACAND", "ATACAND HCT", "AVALIDE", "AVAPRO", "BENICAR", "BENICAR HCT", "COZAAR", "DIOVAN", "DIOVAN HCT", "EDARBI", "EXFORGE", "EXFORGE HCT", "HYZAAR", "MICARDIS", "MICARDIS HCT", "TEVETEN", "TEVETEN HCT" \}

- ATACAND

- ATACAND HCT

- AVALIDE

- AVAPRO

- beNICAR

- benicAR hCt

- cozaAr

- DIOVAN

- DIOVAN HCT

- EDARBI

- EXFORGE

- EXFORGE HCT

- hYZAAR

- MICARDIS

- MICARDIS HCT

- TEVETEN

- TEVETEN HCT

COVID-19 diagnosis:

- This measure creates a logical OR of events, and identifies all occurrences of one or more specified events:

- COVID-19, virus identified, outpatient (U07.1)

- SARS-CoV-2 viral lab test (labs) with positive or presumptive positive result

- Other coronavirus infection (not U07.1)

COVID-19, virus identified: 
medRxiv preprint doi: https://doi.org/10.1101/2020.07.22.20159855; this version posted July 24, 2020. The copyright holder for this preprint (which was not certified by peer review) is the author/funder, who has granted medRxiv a license to display the preprint in perpetuity.

It is made available under a CC-BY-NC-ND 4.0 International license .

- The occurrence of Medical Claims with the following attributes:

- Diagnosis Code, ICD-10 is any of: $\{$ "U07.1" $\}$

- U07.1 - COVID-19, virus identified

COVID-19 viral lab test, with positive of presumed positive result:

- The occurrence of Lab Tests with the following attributes:

- Test Ordered Name is any of: \{ "COVID-19 Nasopharynx", "COVID-19 Oropharynx", "COVID-19 Pooled NP/OP", "SARS CoV 2 RNA(COVID 19), QL NAAT", "SARS Coronavirus with CoV-2 RNA, Quant RT-PCR", "SARS-CoV-2 RNA,QL REAL-TIME RT-PCR (COVID-19)", "SARS-CoV-2 RNA,QL REAL-TIME RT-PCR (COVID-19) - Non-swab", "COVID-19 Nasal/Nasopharynx", "SARS CoV 2 RNA, QL Real Time RT PCR", "SARS CoV 2 RNA, RT-PCR", "SARS-COV-2 RNA (COVID-19), QUALITATIVE NAAT", "SARS-CoV-2 RNA, QL, RT PCR (COVID-19) - Swabs", "SARS-CoV-2 RNA, QL, RT PCR (COVID-19) Swabs", "COVID-19 Pooled N/NP/OP", "SARS CORONAVIRUS WITH COV-2 RNA, QUALITATIVE REAL-TIME RTPCR", "SARS COV 2 RNA, RT PCR", "SARS COV 2 RNA(COVID 19), QUALITATIVE NAAT", "SARS COV W/COV 2 RNA,PCR", "SARS COV 2 RNA, QL REAL TIME RT PCR", "SARS Coronavirus W/CoV 2 RNA, QL Real Time RT PCR", "SARS-CoV-2 RNA, QL Real-Time RT-PCR (CoVID-19) - Non-swab", "SARS CORONAVIRUS W/CoV 2 RNA, QL REAL TIME RT PCR", "SARS-COV-2 RNA, QL REAL-TIME RT-PCR", "SARS-CoV-2 RNA (COVID-19), Qualitative NAAT", "SARS COV 2 RNA, QL NAAT", "SARS Cov 2 RNA, QL NAAT" \}

- COVID-19 Nasopharynx

- COVID-19 Oropharynx

- COVID-19 Pooled NP/OP

- SARS CoV 2 RnA(COVID 19), Ql NAAT

- SARS Coronavirus with CoV-2 RNA, Quant RT-PCR

- SARS-CoV-2 RNA,Ql REAL-TIME RT-PCR (COVID-19)

- SARS-CoV-2 RNA,QL REAL-TIME RT-PCR (COVID-19) - Non-swab

- COVID-19 Nasal/Nasopharynx

- SARs cov 2 RNA, Ql Real Time RT PCR

- sars cov 2 Rna, RT-PCR

- SARS-COV-2 RNA (COVID-19), QUALITATIVE nAAT

- SARS-CoV-2 RNA, QL, RT PCR (COVID-19) - Swabs

- SARS-CoV-2 RNA, QL, RT PCR (COVID-19) Swabs

- COVID-19 Pooled N/NP/OP

- SARS CORONAVIRUS WITH COV-2 RNA, QUALITATIVE REAL-TIME RTPCR

- sars cov 2 rna, rt pCr

- SARS CoV 2 RNA(COVID 19), QUAlitative naAt

- sars cov w/COV 2 RNA,PCR

- SARS cov 2 Rna, Ql REAL time RT PCR

- SARS Coronavirus w/CoV 2 RNA, Ql Real Time RT PCR

- SARS-CoV-2 RNA,QL Real-Time RT-PCR (CoVID-19) - Non-swab

- sars coronavirus W/CoV 2 RNA, Ql REAL tIME RT PCR

- SARS-COV-2 RNA, QL REAL-TIME RT-PCR

- SARS-CoV-2 RNA (COVID-19), Qualitative NAAT

- SARS COV 2 RNA, Ql NAAT

- SARS Cov 2 RNA, QL NAAT

- Result is any of: $\{$ "Positive for 2019-nCoV", "Presumptive Pos. for 2019-nCoV", "Presumptive Positive 2019-nCoV", "Presumptive Positive for 2019-nCoV" \}

- Positive for 2019-nCoV

- Presumptive Pos. for 2019-nCoV

- Presumptive Positive 2019-nCoV

- Presumptive Positive for 2019-nCoV 
medRxiv preprint doi: https://doi.org/10.1101/2020.07.22.20159855; this version posted July 24, 2020. The copyright holder for this preprint (which was not certified by peer review) is the author/funder, who has granted medRxiv a license to display the preprint in perpetuity.

It is made available under a CC-BY-NC-ND 4.0 International license .

- The occurrence of Lab Tests with the following attributes:

- Result Name is any of: \{ "COVID-19 SWB", "SARS CoV 2 RNA, QL Real Time RT PCR", "SARS Coronavirus w/CoV 2 RNA, QL Real Time RT PCR", "COVID-19 Nasopharynx", "SARS-CoV-2 RNA", "SARS-CoV-2 RNA, QL REAL-TIME RT-PCR (COVID-19)", "SARS-COV-2 RNA, QL REAL-TIME RT-PCR (COVID-19) - Non-swab", "COVID-19 Nasal/Nasopharynx", "COVID-19 Oropharynx", "COVID-19 Pooled N/NP/OP", "COVID-19 Pooled NP/OP", "SARS CoV 2 RNA(COVID 19), QL NAAT", "SARS COV 2 RNA:", "SARS COV 2 RNA", "SARS-COV-2 RNA, QL Real-Time RT-PCR (COVID-19) - Non-swab", "SARS COV 2 RNA, RT PCR", "SARS CoV 2 RNA, RT PCR", "SARS-COV-2 RNA:", "SARS-CoV-2 RNA:" \}

- COVID-19 SWB

- SARs cov 2 RnA, Ql Real Time RT PCR

- SARS Coronavirus w/CoV 2 RNA,QL Real Time RT PCR

- COVID-19 Nasopharynx

- SARS-CoV-2 RNA

- SARS-CoV-2 RNA,QL REAL-TIME RT-PCR (COVID-19)

- SARS-CoV-2 RNA,QL REAL-TIME RT-PCR (COVID-19) - Non-swab

- COVID-19 Nasal/Nasopharynx

- COVID-19 Oropharynx

- COVID-19 Pooled N/NP/OP

- COVID-19 Pooled NP/OP

- SARS CoV 2 RnA(COVID 19), Ql NAAT

- SARS CoV 2 RNA:

- sars cov 2 RNA

- SARS-CoV-2 RNA,QL Real-Time RT-PCR (CoVID-19) - Non-swab

- sars cov 2 RnA, RT PCR

- sars Cov 2 RNa, RT PCR

- SARS-COV-2 RNA:

- SARS-CoV-2 RNA:

- Result is any of: \{ "Presumptive Positive for 2019-nCoV", "Presumptive Pos. for 2019-nCoV", "Positive for 2019-nCoV", "Presumptive Positive 2019-nCoV" \}

- Presumptive Positive for 2019-nCoV

- Presumptive Pos. for 2019-nCoV

- Positive for 2019-nCoV

- Presumptive Positive 2019-nCoV

- The occurrence of Lab Tests with the following attributes:

- LOINC Code is any of: $\{$ "943092", "945006" \}

- 943092

- 945006

- Result is any of: \{ "Presumptive Positive for 2019-nCoV", "Presumptive Pos. for 2019-nCoV", "Positive for 2019-nCoV", "Presumptive Positive 2019-nCoV" \}

- Presumptive Positive for 2019-nCoV

- Presumptive Pos. for 2019-nCoV

- Positive for 2019-nCoV

- Presumptive Positive 2019-nCoV

- The occurrence of Lab Tests with the following attributes:

- Procedure Code, HCPCS and CPT is any of: \{ "U0002", "87635", "U0001" \}

- U0002 - Non-CDC laboratory test for SARS-CoV-2/2019-nCoV (COVID-19)

- 87635 - Infectious agent detection by nucleic acid (DNA or RNA); severe acute respiratory syndrome coronavirus 2 (SARS-CoV-2) (Coronavirus disease [COVID-19]), amplified probe technique

- U0001 - CDC laboratory test for SARS-CoV-2/2019-nCoV (COVID-19)

- Result is any of: \{ "Presumptive Positive for 2019-nCoV", "Presumptive Pos. for 2019-nCoV", "Positive for 2019-nCoV", "Presumptive Positive 2019-nCoV" \} 
medRxiv preprint doi: https://doi.org/10.1101/2020.07.22.20159855; this version posted July 24, 2020. The copyright holder for this preprint (which was not certified by peer review) is the author/funder, who has granted medRxiv a license to display the preprint in perpetuity. It is made available under a CC-BY-NC-ND 4.0 International license .

- Presumptive Positive for 2019-nCoV

- Presumptive Pos. for 2019-nCoV

- Positive for 2019-nCoV

- Presumptive Positive 2019-nCoV

Other coronavirus infection (not U07.1):

- This measure creates a logical OR of events, and identifies all occurrences of one or more specified events:

- B34.2 - Coronavirus infection, unspecified

- B97.29 - Other coronavirus as the cause of diseases classified elsewhere

- B97.21 - SARS-associated coronavirus as the cause of diseases classified elsewhere

○ J12.81 - Pneumonia due to SARS-associated coronavirus 
medRxiv preprint doi: https://doi.org/10.1101/2020.07.22.20159855; this version posted July 24, 2020. The copyright holder for this preprint (which was not certified by peer review) is the author/funder, who has granted medRxiv a license to display the preprint in perpetuity.

It is made available under a CC-BY-NC-ND 4.0 International license .

\section{Appendix B: Coding algorithms for outcomes}

\section{Hospitalization with COVID-19 diagnosis:}

- The occurrence of Medical Claims with the following attributes:

- Diagnosis Code, ICD-10 is any of: $\{$ "U07.1" $\}$

- U07.1 - COVID-19, virus identified

- Inpatient Indicator is any of:

- Yes

- The occurrence of Medical Claims with the following attributes:

○ Diagnosis Code, ICD-10 is any of: $\{$ "U07.1" $\}$

- U07.1 - COVID-19, virus identified

- Hospital Service Revenue Code is any of: $\{$ “0012”, “0100”, “0110”, “0116”, "0123”, “0125”, “0129", “0132", “0144", “0145", “0152", “0154", “0159”, “0118”, “0121”, “0124”, “0127”, “0130", “0134", “0136”, “0139”, “0140”, “0150”, “0158”, “0160”, “0167”, “0987”, “0115”, “0117", “0119”, “0133”, “0138”, “0141", “0143”, “0147”, “0148”, “0153”, “0155”, “0164”, “0112", “0113", “0114”, “0120”, “0122”, “0126”, “0128”, “0131”, “0135”, “0151”, “0157”, “0169", "0101", “0111", “0142", “0156” \}

Hospitalization with COVID-19 + the occurrence of ARDS:

This measure was defined as:

- 1.1. The occurrence of Hospitalization with COVID

- 1.2. The occurrence of ARDS, acute respiratory failure, and arrest, starting at least 0 days after the start of 1.1, and starting at most 30 days after the start of 1.1.

- The event of interest in this group is 1.2.

The occurrence of ARDS, acute respiratory failure, and arrest

- The occurrence of Medical Claims with the following attributes:

○ Diagnosis Code, ICD-10 is any of: $\{$ "R09.2", “J80”, “J96.02”, “J96.20”, “J96.21", “J96.22", “J96.00", "J96.2", "J96.0", "J96.01" \}

- R09.2 - Respiratory arrest

- $\quad J 80$ - Acute respiratory distress syndrome

- $\quad 196.02$ - Acute respiratory failure with hypercapnia

- $\quad$ J96.20 - Acute and chronic respiratory failure, unspecified whether with hypoxia or hypercapnia

- $\quad$ J96.21 - Acute and chronic respiratory failure with hypoxia

- $\quad J 96.22$ - Acute and chronic respiratory failure with hypercapnia

- $\quad$ J96.00 - Acute respiratory failure, unspecified whether with hypoxia or hypercapnia

- $\quad$ J96.2 - Acute and chronic respiratory failure

- $\quad$ J96.0 - Acute respiratory failure

- $\quad$ J96.01 - Acute respiratory failure with hypoxia

Hospitalization for COVID-19 + the occurrence of Acute organ dysfunction or failure:

This measure was defined as:

- 1.1. The occurrence of Hospitalization with COVID 
medRxiv preprint doi: https://doi.org/10.1101/2020.07.22.20159855; this version posted July 24, 2020. The copyright holder for this preprint (which was not certified by peer review) is the author/funder, who has granted medRxiv a license to display the preprint in perpetuity.

It is made available under a CC-BY-NC-ND 4.0 International license .

- 1.2. The occurrence of Inpatient hospitalization with acute organ dysfunction or failure, starting at least 0 days after the start of 1.1, and starting at most 30 days after the start of 1.1.

- The event of interest in this group is 1.2 .

The occurrence of Acute organ dysfunction or failure:

- The occurrence of Medical Claims with the following attributes:

- Diagnosis Code, ICD-10 is any of: \{ “G62.81", “N17.9”, “G93.41", "J96.0”, “J96.01", “K72.01”, "N17", “N17.1”, “D65”, “K72.0”, “K72.00", “N17.2", "J96.00”, “J96.02", “N17.8”, “G72.81", “N17.0" \}

- G62.81 - Critical illness polyneuropathy

- N17.9 - Acute kidney failure, unspecified

- G93.41 - Metabolic encephalopathy

- $J 96.0$ - Acute respiratory failure

- $\quad J 96.01$ - Acute respiratory failure with hypoxia

- K72.01 - Acute and subacute hepatic failure with coma

- N17 - Acute kidney failure

- N17.1 - Acute kidney failure with acute cortical necrosis

- D65 - Disseminated intravascular coagulation [defibrination syndrome]

- K72.0 - Acute and subacute hepatic failure

- K72.00 - Acute and subacute hepatic failure without coma

- N17.2 - Acute kidney failure with medullary necrosis

- J96.00 - Acute respiratory failure, unspecified whether with hypoxia or hypercapnia

- J96.02 - Acute respiratory failure with hypercapnia

- N17.8 - Other acute kidney failure

- G72.81 - Critical illness myopathy

- N17.0 - Acute kidney failure with tubular necrosis

- Inpatient Indicator is any of:

- Yes

- The occurrence of Medical Claims with the following attributes:

- Diagnosis Code, ICD-10 is any of: \{ “G62.81", “N17.9”, "G93.41", "J96.0”, “J96.01", “K72.01", “N17”, “N17.1”, “D65”, “K72.0”, “K72.00”, “N17.2”, “J96.00”, “J96.02”, “N17.8”, “G72.81”, "N17.0" \}

- Suspected Inpatient Indicator is any of:

- Yes

Hospitalization with COVID-19 + the occurrence of Sepsis:

This measure was defined as:

- 1.1. The occurrence of Hospitalization with COVID

- 1.2. The occurrence of Occurrence of sepsis inpatient, starting at least 0 days after the start of 1.1, and starting at most 30 days after the start of 1.1.

- The event of interest in this group is 1.2 .

The occurrence of sepsis, inpatient:

- The occurrence of Medical Claims with the following attributes:

O Diagnosis Code, ICD-10 is any of: $\{$ "A02.1", “A26.7”, "A40”, “A40.1”, "A41.01", “A41.1", “A41.59”, “B96.2”, “P35.2”, “A22.7”, “A28.0”, “A32.7”, “A39.4”, “A40.0”, “A40.3”, “A41.52”, “B37.7”, “I76”, “N39.0”, “R65.20”, “A20.7”, “A41”, “A41.02”, “A41.4”, “A41.51”, “A41.89”, “A41.9”, “B00.7”, “B95.61”, “B95.62”, “P36.0”, “P36.2”, “P36.8”, “R65.2”, “A28.2”, “А39.2”, 
medRxiv preprint doi: https://doi.org/10.1101/2020.07.22.20159855; this version posted July 24, 2020. The copyright holder for this preprint (which was not certified by peer review) is the author/funder, who has granted medRxiv a license to display the preprint in perpetuity.

It is made available under a CC-BY-NC-ND 4.0 International license .

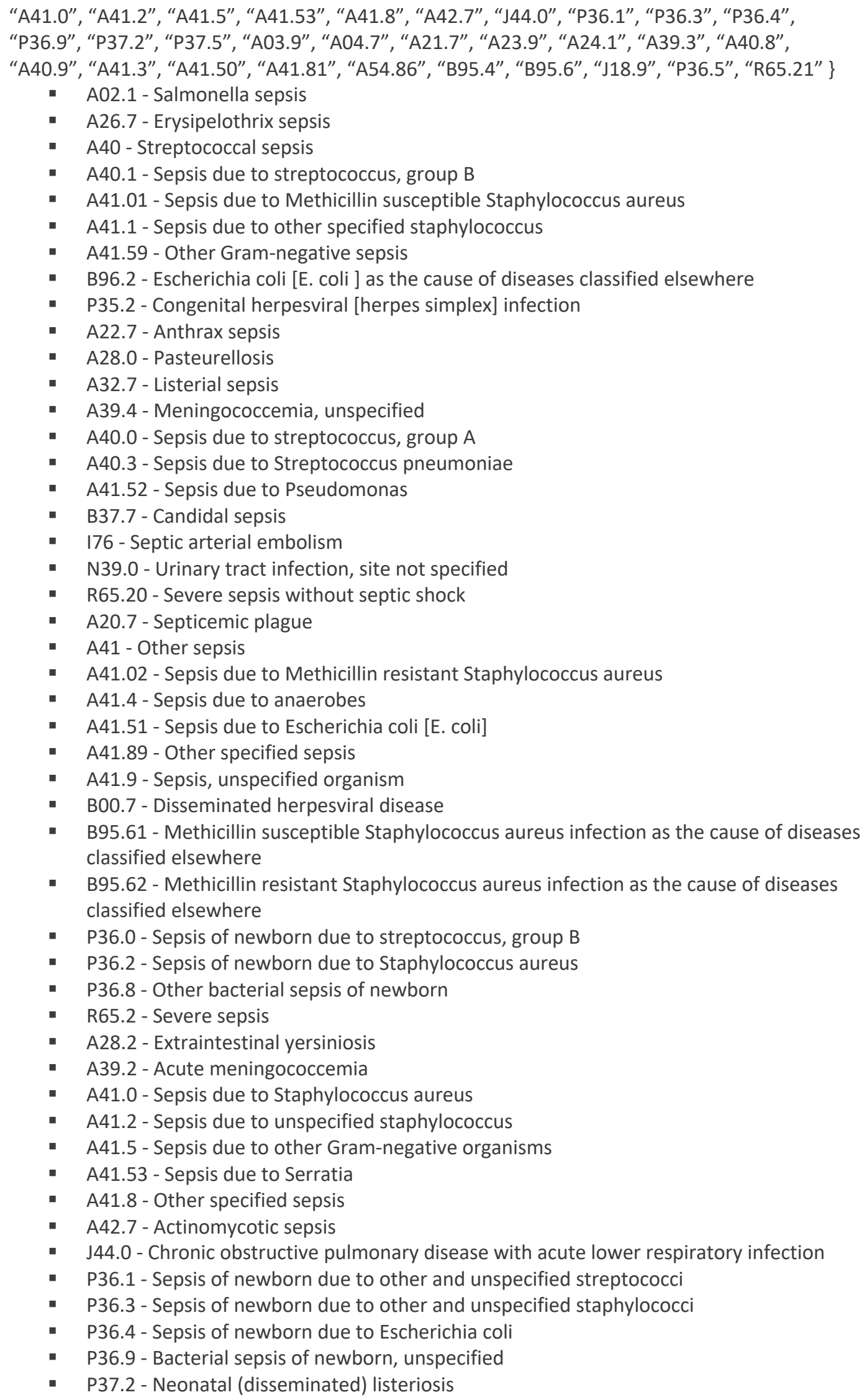


medRxiv preprint doi: https://doi.org/10.1101/2020.07.22.20159855; this version posted July 24, 2020. The copyright holder for this preprint (which was not certified by peer review) is the author/funder, who has granted medRxiv a license to display the preprint in perpetuity. It is made available under a CC-BY-NC-ND 4.0 International license .

- P37.5 - Neonatal candidiasis

- A03.9 - Shigellosis, unspecified

- A04.7 - Enterocolitis due to Clostridium difficile

- A21.7 - Generalized tularemia

- A23.9 - Brucellosis, unspecified

- A24.1 - Acute and fulminating melioidosis

- A39.3 - Chronic meningococcemia

- A40.8 - Other streptococcal sepsis

- A40.9 - Streptococcal sepsis, unspecified

- A41.3 - Sepsis due to Hemophilus influenzae

- A41.50 - Gram-negative sepsis, unspecified

- A41.81 - Sepsis due to Enterococcus

- A54.86 - Gonococcal sepsis

- B95.4 - Other streptococcus as the cause of diseases classified elsewhere

- B95.6 - Staphylococcus aureus as the cause of diseases classified elsewhere

- J18.9 - Pneumonia, unspecified organism

- P36.5 - Sepsis of newborn due to anaerobes

- R65.21 - Severe sepsis with septic shock

- Inpatient Indicator is any of:

- Yes

- The occurrence of Medical Claims with the following attributes:

- Diagnosis Code, ICD-10 is any of: $\{$ "A02.1", "A26.7", "A40", "A40.1", "A41.01", "A41.1", “A41.59", “B96.2", “P35.2", “A22.7”, “A28.0”, “A32.7”, “A39.4”, “A40.0”, “A40.3”, “A41.52", "B37.7", “176", “N39.0", “R65.20”, “A20.7", “A41", “A41.02", “A41.4”, “A41.51", “A41.89”, “A41.9”, “B00.7”, “B95.61", “B95.62", “P36.0”, “P36.2”, “P36.8”, “R65.2”, “A28.2”, “A39.2”, "A41.0”, “A41.2”, “A41.5”, “A41.53”, “A41.8”, “A42.7”, “J44.0”, “P36.1”, “P36.3”, “P36.4”, “P36.9”, “P37.2”, “P37.5”, “A03.9”, “A04.7”, “A21.7”, “A23.9”, “A24.1”, “A39.3”, “A40.8”,

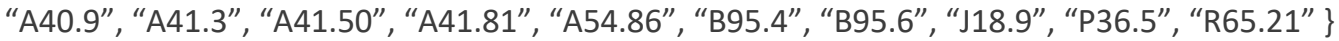

- Suspected Inpatient Indicator is any of:

- Yes

- The occurrence of Medical Claims with the following attributes:

- Diagnosis Code, ICD-10 is any of: $\{$ "A02.1", "A26.7", "A40", "A40.1", "A41.01", "A41.1",



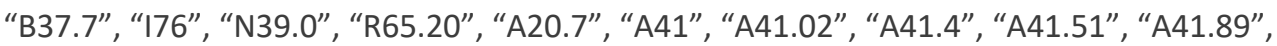
"A41.9”, “B00.7”, “B95.61", “B95.62", “P36.0”, “P36.2”, “P36.8”, “R65.2”, “A28.2”, “А39.2”, "A41.0", “A41.2", “A41.5”, “A41.53", “A41.8”, “A42.7”, “J44.0”, “P36.1”, “P36.3”, “P36.4”, “P36.9”, “P37.2", “P37.5”, “A03.9”, “A04.7”, “A21.7”, “A23.9”, “A24.1”, “A39.3”, “A40.8”, "A40.9”, “A41.3", “A41.50", “A41.81", “A54.86", “B95.4”, “B95.6”, “J18.9”, “P36.5”, “R65.21” \}

○ Hospital Service Revenue Code is any of: \{ “0114", "0115", “0129", “0142", “0143", "0144", “0151", “0156", “0987", “0100", “0101", “0118”, “0122", “0127", “0133", “0152", “0160”, “0117", “0120", “0130", “0136", “0140", “0145", “0150", “0155", “0158”, “0169”, “0012", “0111", “0113", “0116", “0119”, “0121", “0125", “0139”, “0141", “0147", “0148”, “0154”, “0157", “0164", “0167", “0110”, “0112", “0123”, “0124”, “0126”, “0128”, “0131”, “0132", “0134", “0135”, “0138”, “0153”, “0159” \} 
medRxiv preprint doi: https://doi.org/10.1101/2020.07.22.20159855; this version posted July 24, 2020. The copyright holder for this preprint

(which was not certified by peer review) is the author/funder, who has granted medRxiv a license to display the preprint in perpetuity.

It is made available under a CC-BY-NC-ND 4.0 International license .

Hospitalization with COVID-19 + the occurrence of respiratory intubation and mechanical ventilation (procedure and diagnosis codes):

This measure was defined as:

- 1.1. The occurrence of Hospitalization with COVID

- 1.2. The occurrence of Respiratory intubation and mechanical ventilation (procedure and diagnosis codes), starting at least 0 days after the start of 1.1 , and starting at most 30 days after the start of 1.1.

- The event of interest in this group is 1.2.

The occurrence of respiratory intubation and mechanical ventilation (procedure and diagnosis codes):

- The occurrence of Medical Claims with the following attributes:

- Procedure Code, HCPCS and CPT is any of: \{ "31500", "G8569", "43753", "94002", "94657", "94003", "94004", "94656" \}

- 31500 - Intubation, endotracheal, emergency procedure

- G8569 - PROLONGED POSTOPERATIVE INTUBATION (> 24 HRS) REQUIRED

- 43753 - Gastric intubation and aspiration(s) therapeutic, necessitating physician's skill (eg, for gastrointestinal hemorrhage), including lavage if performed

- 94002 - Ventilation assist and management, initiation of pressure or volume preset ventilators for assisted or controlled breathing; hospital inpatient/observation, initial day

- 94657 - Ventilation assist and management, initiation of pressure or volume preset ventilators for assisted or controlled breathing; subsequent days

- 94003 - Ventilation assist and management, initiation of pressure or volume preset ventilators for assisted or controlled breathing; hospital inpatient/observation, each subsequent day

- 94004 - Ventilation assist and management, initiation of pressure or volume preset ventilators for assisted or controlled breathing; nursing facility, per day

- 94656 - Ventilation assist and management, initiation of pressure or volume preset ventilators for assisted or controlled breathing; first day

- The occurrence of Medical Claims with the following attributes:

○ Diagnosis Code, ICD-10 is any of: \{ "Z99.1", "Z99.11", "Z99.12", "J95.851", "J95.85", "J95.859",

"T88.4", "T88.4XXA", "T88.4XXS" \}

- Z99.1 - Dependence on respirator

- Z99.11 - Dependence on respirator [ventilator] status

- Z99.12 - Encounter for respirator [ventilator] dependence during power failure

- $\quad J 95.851$ - Ventilator associated pneumonia

- $\quad J 95.85$ - Complication of respirator [ventilator]

- J95.859 - Other complication of respirator [ventilator]

- T88.4 - Failed or difficult intubation

- T88.4XXA - Failed or difficult intubation, initial encounter

- T88.4XXS - Failed or difficult intubation, sequela

- The occurrence of Medical Claims with the following attributes:

- Procedure Code, ICD-10 is any of: \{ "09HN8BZ", "OBH18EZ", "ODH57BZ", "OWHQ73Z", "OWHQ7YZ", “5A09457”, “09HN7BZ", “5A09557”, “OCHY7BZ", “5A09357”, “5A1945Z”, “5A1955Z”, “OBH17EZ”, “OBH13EZ”, “OCHY8BZ”, “ODH58BZ”, “5A1935Z” \}

- $09 H$ H8BZ - Insertion of Airway into Nasopharynx, Via Natural or Artificial Opening Endoscopic

- OBH18EZ - Insertion of Endotracheal Airway into Trachea, Via Natural or Artificial Opening Endoscopic

- ODH57BZ - Insertion of Airway into Esophagus, Via Natural or Artificial Opening 
medRxiv preprint doi: https://doi.org/10.1101/2020.07.22.20159855; this version posted July 24, 2020. The copyright holder for this preprint

(which was not certified by peer review) is the author/funder, who has granted medRxiv a license to display the preprint in perpetuity.

It is made available under a CC-BY-NC-ND 4.0 International license .

- OWHQ73Z - Insertion of Infusion Device into Respiratory Tract, Via Natural or Artificial Opening

- OWHQ7YZ - Insertion of Other Device into Respiratory Tract, Via Natural or Artificial Opening

- 5A09457 - Assistance with Respiratory Ventilation, 24-96 Consecutive Hours, Continuous Positive Airway Pressure

- $09 H$ N7BZ - Insertion of Airway into Nasopharynx, Via Natural or Artificial Opening

- 5A09557 - Assistance with Respiratory Ventilation, Greater than 96 Consecutive Hours, Continuous Positive Airway Pressure

- $\quad$ OCHY7BZ - Insertion of Airway into Mouth and Throat, Via Natural or Artificial Opening

- 5A09357 - Assistance with Respiratory Ventilation, Less than 24 Consecutive Hours, Continuous Positive Airway Pressure

- 5A1945Z - Respiratory Ventilation, 24-96 Consecutive Hours

- 5A1955Z - Respiratory Ventilation, Greater than 96 Consecutive Hours

- OBH17EZ - Insertion of Endotracheal Airway into Trachea, Via Natural or Artificial Opening

- OBH13EZ - Insertion of Endotracheal Airway into Trachea, Percutaneous Approach

- OCHY8BZ - Insertion of Airway into Mouth and Throat, Via Natural or Artificial Opening Endoscopic

- $\quad$ ODH58BZ - Insertion of Airway into Esophagus, Via Natural or Artificial Opening Endoscopic

- 5A1935Z - Respiratory Ventilation, Less than 24 Consecutive Hours 